Electronic Journal of Statistics

Vol. 15 (2021) 3708-3742

ISSN: $1935-7524$

https://doi.org/10.1214/21-EJS1874

\title{
A simulation-extrapolation approach for the mixture cure model with mismeasured covariates*
}

\author{
Eni Musta ${ }^{1,2}$ and Ingrid Van Keilegom ${ }^{1}$ \\ ${ }^{1}$ ORSTAT, KU Leuven, Belgium \\ e-mail: ingrid.vankeilegom@kuleuven.be \\ ${ }^{2}$ Korteweg de Vries Institute for Mathematics, University of Amsterdam, Netherlands \\ e-mail: e.musta@uva.nl
}

\begin{abstract}
We consider survival data from a population with cured subjects in the presence of mismeasured covariates. We use the mixture cure model to account for the individuals that will never experience the event and at the same time distinguish between the effect of the covariates on the cure probabilities and on survival times. In particular, for practical applications, it seems of interest to assume a logistic form of the incidence and a Cox proportional hazards model for the latency. To correct the estimators for the bias introduced by the measurement error, we use the simex algorithm, which is a very general simulation based method. It essentially estimates this bias by introducing additional error to the data and then recovers bias corrected estimators through an extrapolation approach. The estimators are shown to be consistent and asymptotically normally distributed when the true extrapolation function is known. We investigate their finite sample performance through a simulation study and apply the proposed method to analyse the effect of the prostate specific antigen (PSA) on patients with prostate cancer.
\end{abstract}

MSC2020 subject classifications: Primary $62 \mathrm{~N} 02$.

Keywords and phrases: Cure models, logistic model, measurement error, simex algorithm, survival analysis.

Received November 2020.

\section{Introduction}

Classical survival analysis methods are designed to deal with time-to-event data in the presence of censoring and covariates. However, they often fail to address various challenges presented by real-life problems. In recent times, significant advances have been made in adapting and extending traditional methods for handling data with more complex features. In this article we account simultaneously for a cure fraction of the population, referring to those subjects that are immune to the event of interest, and covariates measured with error. Such situations arise frequently in practice. For instance, in cancer studies it is known that

* The authors acknowledge financial support from the European Research Council (20162021, Horizon 2020, grant agreement 694409). For the simulations we used the infrastructure of the Flemish Supercomputer Center (VSC). 
some of the patients will never experience recurrence or cancer related death and certain biomarker expressions such as the hemoglobin level or tumor size cannot be measured precisely. The systolic blood pressure is also known to be an error-prone predictor for the development of the coronary heart disease. Examples of variables that cannot be measured precisely end events that are not experienced by the whole population can also be found in economic and social studies. Ignoring both these characteristics in the statistical procedures would most probably lead to incorrect inferences.

Cure rate models were first introduced in [7] and [3], but only quite recently they have attracted attention in the statistical literature and applications. The proposed models can be divided in two main categories: mixture cure models and promotion time models (see [1] for a detailed review). The first ones assume that the population consists of two subpopulations, the cured and the susceptible ones, and model separately the incidence (the probability of being noncured) and the latency (the survival of the noncured subjects) using parametric or nonparametric models. The latter ones have a proportional hazards structure and extend the classical Cox regression model to allow for the survival function to flatten at a level greater than zero. There is no clear indication of which approach is more appropriate but in general, mixture cure models are preferred when one wants to distinguish between variables that affect the cure probability and the survival of the uncured subjects.

On the other hand, there is a vast literature about bias correction methods mainly in regression models with covariates contaminated by measurement error ([11]). The classical additive error model is generally accepted and the most common methods to deal with it are the so called functional ones, which do not make any assumption on the distribution of the unobserved true covariates. They can be divided in three large classes of models: regression calibration, score functional methods and simulation-extrapolation (simex). Regression calibration is a computationally simple method which replaces the mismeasured covariate by its conditional mean given the observed variables. However, for estimation of this conditional mean, a parametric model is assumed and replicated measurements or validation data are required ([11, 37]). The corrected score approach is mathematically more involved since it constructs the estimators as $M$-estimators based on a corrected score function that is unbiased in the presence of the measurement error. As a result, this method depends on specific model assumptions and cannot be easily extended or adapted to variations of the model $([33,27])$. The simex approach is quite appealing because it is a simulation based method and it can be easily adapted to any kind of model. It only requires an estimation method in the absence of measurement error and can be easily implemented (though computationally more intensive). In survival analysis it has been applied to the semiparametric Cox model ([11]), the marginal hazards model for multivariate failure time data ([16]), the frailty model for clustered survival data ([20]) etc.

However, there are only limited studies on cure rate models with measurement error. This problem was first addressed in [24] and [23], who propose a corrected score approach for the parametric and semiparametric promotion time 
models respectively. Afterwards, the simex procedure was introduced as an alternative estimation method in a more general version of promotion time models in [4] and an extensive simulation study was done in [5] to compare it with the corrected score approach and get a better understanding on the robustness of the method. In the context of mixture cure models, the simex algorithm has only been proposed for left-truncated right-censored data when a transformation model is assumed for the latency ([12]). However, [12] considers only the case in which the mismeasured covariate affects only the latency and theory is developed for one specific estimation method based on martingale integral representations. In particular, the most commonly used logistic/Cox mixture cure model for right-censored data and the maximum likelihood estimation method (based on the EM algorithm) have not been investigated in presence of measurement error. The popularity of this model motivates us to search for solutions to correct estimates for the biases induced by the measurement error.

Here we propose a simex approach for a general mixture cure model with a parametric form of the incidence and a semiparametric model for the latency. Any estimation method in the absence of measurement error can be used within the simex algorithm. We focus mainly on the logistic/Cox setting, given its practical relevance, but the proposed procedure and the asymptotic theory hold for other mixture cure models as well, provided that the considered estimation method in the absence of measurement error satisfies certain conditions. In particular, these conditions are satisfied for the maximum likelihood estimator introduced in [35] and the presmoothing approach proposed in [26]. We use both these estimators in the simex procedure and compare them through a simulation study. In contrast to the previously considered promotion time models, here we find that if the mismeasured covariate affects only one of the two components (incidence or latency), the estimation of the other component remains undisturbed even if the variables are correlated.

The article is organized as follows. We start by describing a general parametric/ semiparametric mixture cure model with measurement error in Section 2 and then explain the simex estimation procedure in Section 3. Asymptotic properties of the estimators are presented in Section 4, while their practical performance for the logistic/Cox mixture cure model is demonstrated through simulation studies in Section 5. Finally, in Section 6, we apply the proposed method to a prostate cancer dataset to account for measurement error in the values of the prostate specific antigen. Proofs and additional simulation results are presented in the Appendix.

\section{Mixture cure model with measurement error}

Suppose we are interested in the time $T$ until a certain event happens. In contrast to classical survival analysis, in cure models it is possible to have $T=\infty$ (the event never happens), reflecting the presence of a cure fraction. On the other hand, a finite survival time corresponds to susceptible subjects that will experience the event at some time point. If we indicate by $B$ the uncured status, 
i.e. $B=\mathbb{1}_{\{T<\infty\}}$, then we can write

$$
T=B T_{0}+(1-B) \infty,
$$

with $T_{0}$ representing the survival time for an uncured individual. The challenge of dealing with this type of models arises from the fact that, because of finite censoring times, it is impossible to completely separate the two groups. To be precise, if $C$ denotes the censoring time, then we only observe the follow-up time $Y=\min (T, C)$ and the censoring indicator $\Delta=\mathbb{1}_{\{T<C\}}$. Hence, for the observations with $\Delta=0$, we do not know whether they are cured or susceptible. In addition to the cure fraction and censoring, it is desirable to also account for the impact of certain covariates on the time to event variable. Let $\left(X^{T}, Z^{T}\right)^{T}$ a $(p+q)$-dimensional vector of covariates, where $x^{T}$ denotes the transpose of the vector $x$. The advantage of mixture cure models with respect to promotion time models is that they can distinguish between the covariates $X$, which affect the cure rate, and $Z$, which affect the survival of the uncured subjects, i.e.

$$
\mathbb{P}(T=\infty \mid X, Z)=\mathbb{P}(T=\infty \mid X) \quad \text { and } \quad \mathbb{P}(T<\infty \mid X, Z)=\mathbb{P}(T<\infty \mid Z) .
$$

However, it is possible for $X$ and $Z$ to be the same or share some of the components. As commonly done in studies of cure models, we assume that the censoring time and the survival time are independent given the covariates

$$
T \perp C \mid(X, Z),
$$

which is equivalent to requiring $T_{0} \perp(C, X) \mid Z$ and $B \perp\left(C, T_{0}, Z\right) \mid X$ (see Lemma 1 in Appendix A of [26]).

In this paper we deal with situations in which some of the continuous covariates included in $X$ and/or $Z$ are subject to measurement error. For ease of notation and interpretation we define the vector of unique covariates

$$
\left(E^{(1)^{T}}, E^{(2)^{T}}, E^{(3)^{T}}\right)^{T} \in \mathbb{R}^{p+q_{1}},
$$

where $E^{(1)}$ denotes the covariates in $X$ that are not present in $Z, E^{(2)}$ denotes the common components of $X$ and $Z, E^{(3)}$ denotes the covariates in $Z$ that are not present in $X$ and $q_{1}$ is the number of covariates in $E^{(3)}$. In other words, we are removing the repeated covariates from the vector $\left(X^{T}, Z^{T}\right)^{T}$ without loosing any information. In the presence of measurement error, instead of $\left(E^{(1)^{T}}, E^{(2)^{T}}, E^{(3)^{T}}\right)^{T}$, we observe $W=\left(W^{(1)^{T}}, W^{(2)^{T}}, W^{(3)^{T}}\right)^{T}$ such that

$$
W=\left(E^{(1)^{T}}, E^{(2)^{T}}, E^{(3)^{T}}\right)^{T}+U
$$

where $U \in \mathbb{R}^{p+q_{1}}$ is the vector of measurement errors. We assume that $U$ is independent of $(X, Z, T, C)$ and it follows a continuous distribution with mean zero and known variance matrix $V$. The elements of $V$ corresponding to covariates with no measurement error (including non-continuous covariates) are set to zero. However, no parametric assumption is made on the distribution of the errors. In particular, the measurement error is not required to be normally distributed. 
We consider a general mixture cure model with a parametric form of the incidence and a semiparametric model for the latency. To be precise, the cure probability of a subject with covariate $x$ is

$$
\pi_{0}(x)=1-\phi\left(\gamma^{*}, x\right)
$$

for some known function $\phi: \mathbb{R}^{p} \times \mathbb{R}^{p} \mapsto[0,1]$ and $\gamma^{*} \in \mathbb{R}^{p}$, while the conditional survival function of the noncured subjects $S_{u}(\cdot \mid z)$ depends on a parametric component $\beta^{*}$ and a nonparametric non-decreasing function $\Lambda^{*}$ (for example the cumulative baseline hazard). As a result, the conditional survival function corresponding to $T$ is then

$$
S(t \mid x, z)=\mathbb{P}(T>t \mid X=x, Z=z)=1-\phi\left(\gamma^{*}, x\right)+\phi\left(\gamma^{*}, x\right) S_{u}(t \mid z) .
$$

Choosing a parametric model for the incidence seems quite standard in the literature of mixture cure models $([29,8,32])$ because of its simplicity and ease of interpretability (particularly for multiple covariates). Recently there have been also a few nonparametric proposals $([39,2,21])$ but we choose to focus on the previous models since they are more widely used in practice $([18,40,38,34])$. In addition, nonparametric estimation of $\pi_{0}(\cdot)$ would require repeating the simex procedure for multiple points of the support, which is very computationally intensive. In general, it is possible to test whether this assumption is reasonable ([25]). Among the parametric models for the incidence component, the logistic model where

$$
\phi(\gamma, x)=\frac{e^{\gamma^{T} x}}{1+e^{\gamma^{T} x}},
$$

is perhaps the most common one. On the other hand, the Cox proportional hazards model ([14])

$$
S_{u}(t \mid z)=\exp \left\{-\Lambda^{*}(t) \exp \left(\beta^{* T} z\right)\right\},
$$

and the accelerated failure time model

$$
S_{u}(t \mid z)=\exp \left\{-\Lambda^{*}\left(\exp \left(\beta^{* T} z\right) t\right)\right\},
$$

where $\Lambda^{*}$ is the baseline cumulative hazard function, are both widely used semiparametric modelling approaches for the latency $([30,35,19])$. However, our methodology applies more in general to parametric/semiparametric mixture cure models provided that an estimation method for the case without measurement error is available. The goal is to estimate the true parameters $\gamma^{*}$, $\beta^{*}$ and $\Lambda^{*}$ on the basis of $n$ i.i.d. observations $\left(Y_{1}, \Delta_{1}, W_{1}\right), \ldots,\left(Y_{n}, \Delta_{n}, W_{n}\right)$, knowing the variance matrix $V$ of the measurement error. In the next section we propose a simulation-extrapolation approach designed to reduce the bias due to the measurement error.

\section{Methodology}

The basic idea behind the simex algorithm is that we can gain insights on how the measurement error affects the estimators by creating artificial data 
with increasing levels of measurement error and estimating the parameters as if there was no error. The obtained information is then used in the second step to recover the bias corrected estimators through an extrapolation approach. Next we describe the details of this procedure.

Step 1. (Simulation) We choose $K$ levels of added noise $\lambda_{1}, \ldots, \lambda_{K} \geq 0$ and for each of them we generate a large number $B$ of artificially contaminated samples. To be precise, for each $\lambda \in\left\{\lambda_{1}, \ldots, \lambda_{K}\right\}$ and $b \in\{1, \ldots, B\}$, we simulate independent identically distributed variables $\left\{\tilde{U}_{b, i}\right\}_{i=1}^{n}$, independently of the observed data and with distribution $N_{D}\left(0, I_{D}\right)$, where $D=p+q_{1}$ is the dimension of the vector $W$. Afterwards, we construct new covariates

$$
W_{i, \lambda, b}=W_{i}+(\lambda V)^{1 / 2} \tilde{U}_{i, b},
$$

where $V$ is the covariance matrix of the error in (2). Distributions different from Gaussian can be used too but here we focus on normal errors. The mixture model satisfied by the new covariates $W_{i, \lambda, b}$

$$
\begin{aligned}
& S\left(t \mid\left(W_{i, \lambda, b}^{(1)}, W_{i, \lambda, b}^{(2)}\right),\left(W_{i, \lambda, b}^{(2)}, W_{i, \lambda, b}^{(3)}\right)\right) \\
& =1-\phi\left(\gamma_{\lambda},\left(W_{i, \lambda, b}^{(1)}, W_{i, \lambda, b}^{(2)}\right)\right)+\phi\left(\gamma_{\lambda},\left(W_{i, \lambda, b}^{(1)}, W_{i, \lambda, b}^{(2)}\right)\right) S_{u, \lambda}\left(t \mid\left(W_{i, \lambda, b}^{(2)}, W_{i, \lambda, b}^{(3)}\right)\right),
\end{aligned}
$$

is characterized by the parameters $\gamma_{\lambda}, \beta_{\lambda}$ and $\Lambda_{\lambda}$. Using $\left\{Y_{i}, \Delta_{i}, W_{i, \lambda, b}\right\}_{i=1}^{n}$ we estimate $\gamma_{\lambda}, \beta_{\lambda}$ and $\Lambda_{\lambda}$, as if there was no measurement error, obtaining $\hat{\gamma}_{\lambda, b}, \hat{\beta}_{\lambda, b}$ and $\hat{\Lambda}_{\lambda, b}$. The latter one is an estimator of $\Lambda^{*}$ over some compact interval $[0, \tau]$. Any available estimation method can be used. For example, in the logistic/Cox mixture cure model, the maximum likelihood estimation ([35, 9]) or the presmoothing approach proposed in [26] can be considered.

At the end, for each level of contamination, the average values of all the $B$ estimates are calculated:

$$
\hat{\gamma}_{\lambda}=\frac{1}{B} \sum_{b=1}^{B} \hat{\gamma}_{\lambda, b}, \quad \hat{\beta}_{\lambda}=\frac{1}{B} \sum_{b=1}^{B} \hat{\beta}_{\lambda, b} \quad \text { and } \quad \hat{\Lambda}_{\lambda}(t)=\frac{1}{B} \sum_{b=1}^{B} \hat{\Lambda}_{\lambda, b}(t) .
$$

Note that, if the estimators $\hat{\Lambda}_{\lambda, b}$ are piecewise constant with jumps at the observed event times, then also $\hat{\Lambda}_{\lambda}$ is piecewise constant with jumps at the observed event times. The parameters to be chosen in this step are $K$, the $\lambda$ values and $B$. Common values are $K=5, \lambda \in\{0,0.5,1,1.5,2\}$ and $B=50([10,13])$.

Step 2. (Extrapolation) Note that, by independence, the covariance matrix of the simulated covariates $W_{i, \lambda, b}$ is

$$
\operatorname{var}\left(W_{i, \lambda, b} \mid X_{i}\right)=\operatorname{var}\left(W_{i} \mid X_{i}\right)+\lambda V=(1+\lambda) V .
$$

This means that the variance has been inflated by a factor $1+\lambda$ and that the ideal case of no measurement error corresponds to $\lambda=-1$ (adding 'negative' variance). Hence, the idea is to model the relationship between $\lambda$ and the estimators $\hat{\gamma}_{\lambda}, \hat{\beta}_{\lambda}, \hat{\Lambda}_{\lambda}$ by fitting a regression function and then extrapolate to 
$\lambda=-1$. First, an extrapolant function needs to be chosen (e.g. linear, quadratic or fractional) for each component of $\hat{\gamma}_{\lambda}, \hat{\beta}_{\lambda}, \hat{\Lambda}_{\lambda}$ as a function of $\lambda$. For example, for the quadratic case and $\lambda \in\left\{\lambda_{1}, \ldots, \lambda_{K}\right\}$, we have

$$
\begin{aligned}
& \hat{\gamma}_{\lambda, j}=g_{\gamma, j}\left(a_{\gamma_{j}}^{*}, \lambda\right)+\epsilon_{\gamma, \lambda, j}=a_{\gamma_{j}, 1}^{*}+a_{\gamma_{j}, 2}^{*} \lambda+a_{\gamma_{j}, 3}^{*} \lambda^{2}+\epsilon_{\gamma, \lambda, j}, \quad j=1, \ldots, p \\
& \hat{\beta}_{\lambda, j}=g_{\beta, j}\left(a_{\beta_{j}}^{*}, \lambda\right)+\epsilon_{\beta, \lambda, j}=a_{\beta_{j}, 1}^{*}+a_{\beta_{j}, 2}^{*} \lambda+a_{\beta_{j}, 3}^{*} \lambda^{2}+\epsilon_{\beta, \lambda, j}, \quad j=1, \ldots, q \\
& \hat{\Lambda}_{\lambda}(t)=g_{\Lambda, t}\left(a_{t}^{*}, \lambda\right)+\epsilon_{\Lambda, \lambda, t}=a_{t, 1}^{*}+a_{t, 2}^{*} \lambda+a_{t, 3}^{*} \lambda^{2}+\epsilon_{\Lambda, \lambda, t}, \quad t \in[0, \tau],
\end{aligned}
$$

where $\epsilon_{\beta, \lambda, j}, \epsilon_{\gamma, \lambda, j}$ and $\epsilon_{\Lambda, \lambda, t}$ are the error terms in the extrapolant model, assumed to have mean zero and to be independent. We obtain estimators $\hat{a}_{\gamma_{j}}=$ $\left(\hat{a}_{\gamma_{j}, 1}, \hat{a}_{\gamma_{j}, 2}, \hat{a}_{\gamma_{j}, 3}\right), \hat{a}_{\beta_{j}}=\left(\hat{a}_{\beta_{j}, 1}, \hat{a}_{\beta_{j}, 2}, \hat{a}_{\beta_{j}, 3}\right)$ and $\hat{a}_{t}=\left(\hat{a}_{t, 1}, \hat{a}_{t, 2}, \hat{a}_{t, 3}\right)$ of the unknown parameters of the extrapolant function by fitting the previous regression models using the method of least squares. Finally, the simex estimators are defined by

$$
\begin{array}{ll}
\hat{\gamma}_{j, \text { simex }}=\lim _{\lambda \rightarrow-1} g_{\gamma, j}\left(\hat{a}_{\gamma_{j}}, \lambda\right), & j=1, \ldots, p, \\
\hat{\beta}_{j, \text { simex }}=\lim _{\lambda \rightarrow-1} g_{\beta, j}\left(\hat{a}_{\beta_{j}}, \lambda\right), & j=1, \ldots, q, \\
\hat{\Lambda}_{\text {simex }}(t)=\lim _{\lambda \rightarrow-1} g_{\Lambda, t}\left(\hat{a}_{t}, \lambda\right), & t \in[0, \tau] .
\end{array}
$$

If the initial estimators $\hat{\Lambda}_{\lambda, b}$ are piecewise constant with jumps at the observed event times, then the extrapolation procedure needs to be applied only for the observed event times $t \in\left\{T_{(1)}, \ldots, T_{(m)}\right\}$. Equivalently, the procedure can be applied to the jump sizes for different coefficients $a^{*}$ and a possibly different extrapolation function (if it is not polynomial). Even though this does not guarantee that the resulting estimator $\hat{\Lambda}_{\text {simex }}$ is non-decreasing, in practice this is often the case. If one is interested in estimation of $\Lambda^{*}$ on the whole support and $\hat{\Lambda}_{\text {simex }}$ is not monotone, an isotonized version of it, using for example the pool-adjacent-violators algorithm ([31]), would be a more reasonable estimate. However, here we focus on estimation of the parameters $\gamma, \beta$ and do not further exploit this aspect. Note also that different extrapolation functions lead to different results. Hence it is important to have a good approximation of the true extrapolation function.

\section{Asymptotic properties}

\subsection{General results}

In this section we will discuss the identifiability of the model and establish some theoretical results regarding the large-sample properties of the proposed estimators. A drawback of the simex approach is that consistency and asymptotic normality of the estimators hold only if we knew the true extrapolation function, which is usually not the case in practice. When the true extrapolant function is not known, but an approximation of it is used, the results 
of Theorems 1 and 2 hold with $\gamma^{*}, \beta^{*}, \Lambda^{*}(t)$ replaced by $\lim _{\lambda \rightarrow-1} g_{\gamma}\left(a_{\gamma}, \lambda\right)$, $\lim _{\lambda \rightarrow-1} g_{\beta}\left(a_{\beta}, \lambda\right)$ and $\lim _{\lambda \rightarrow-1} g_{\Lambda}\left(a_{t}, \lambda\right)$ respectively. Here $g_{\gamma}\left(a_{\gamma}, \lambda\right)$ denotes the vector $\left(g_{\gamma, 1}\left(a_{\gamma_{1}}, \lambda\right), \ldots, g_{\gamma, p}\left(a_{\gamma_{p}}, \lambda\right)\right)^{T}$ and $g_{\beta}\left(a_{\beta}, \lambda\right), g_{\Lambda}\left(a_{t}, \lambda\right)$ are defined similarly. We first establish the asymptotic results in a general mixture cure model as described in Section 2, assuming that the used estimation method for obtaining $\hat{\gamma}_{\lambda, b}, \hat{\beta}_{\lambda, b}, \hat{\Lambda}_{\lambda, b}$ (ignoring the measurement error) satisfies certain conditions. Afterwards, we will focus on two estimation methods for the logistic/Cox mixture cure model and show that the required conditions are met. All the proofs can be found in the Appendix.

For a fixed $\lambda>0$ consider observations $\left(Y, \Delta, W_{\lambda}\right)$, where $W_{\lambda}=W+$ $(\lambda V)^{1 / 2} \tilde{U}$ and the mixture cure model with conditional survival

$S\left(t \mid W_{\lambda}\right)=1-\phi\left(\gamma_{\lambda},\left(W_{\lambda}^{(1)}, W_{\lambda}^{(2)}\right)\right)+\phi\left(\gamma_{\lambda},\left(W_{\lambda}^{(1)}, W_{\lambda}^{(2)}\right)\right) S_{u, \lambda}\left(t \mid\left(W_{\lambda}^{(2)}, W_{\lambda}^{(3)}\right)\right)$,

where, as mentioned in Section 2, the decomposition of $W_{\lambda}$ in three components corresponds to the covariates that influence only the cure probability, those that are common for the incidence and the latency and the ones that affect only the latency. The survival of the uncured subject $S_{u, \lambda}$ depends on the regression parameters $\beta_{\lambda}$ and the nonparametric function $\Lambda_{\lambda}$. Suppose we have an estimation method that provides estimates $\hat{\gamma}_{\lambda}, \hat{\beta}_{\lambda}$ and $\hat{\Lambda}_{\lambda}$, the latter one being a non-decreasing function.

The mixture cure model in (3) is identifiable when the following basic conditions are satisfied:

(I1) if $\phi(\gamma, X)=\phi(\tilde{\gamma}, X)$ almost surely, then $\gamma=\tilde{\gamma}$,

(I2) the function $S_{u}(\cdot \mid z)$ has support $[0, \tau(z)]$,

(I3) $\mathbb{P}(C>\tau(Z) \mid X, Z)>0$ for almost all $X$ and $Z$,

(I4) if, for all $t \geq 0$, we have $S_{u}(t \mid Z ; \Lambda, \beta)=S_{u}(t \mid Z ; \tilde{\Lambda}, \tilde{\beta})$ almost surely, then $\Lambda=\tilde{\Lambda}$ and $\beta=\tilde{\beta}$,

where identifiability means that different parameter values lead to different distributions of the observed variables $(Y, \Delta, X, Z)$. This can be proved in the same way as in [28] (or as Lemma 1 in [2]). In the particular case of the logistic-Cox model the conditions become:

(I1') for all $x, z, 0<\phi(\gamma, x)<1$,

(I2') the function $S_{u}$ has support $\left[0 ; \tau_{0}\right]$ for some $\tau_{0}<\infty$,

(I3') $P\left(C>\tau_{0} \mid X ; Z\right)>0$ for almost all $\mathrm{X}$ and $\mathrm{Z}$,

(I4') the matrices $\operatorname{Var}(X)$ and $\operatorname{Var}(Z)$ are positive definite,

(see Proposition 1 and 2 in [28]). When these conditions are satisfied by the original model, they are also satisfied for the model in (8) with the mismeasured covariates because of the assumptions on $W, \tilde{U}, V$ (the matrix $\operatorname{Var}\left(W_{\lambda}\right)$ is positive definite for all $\lambda>0$ ). Moreover, for known extrapolation functions, the parameters $\gamma^{*}, \beta^{*}$ and $\Lambda^{*}$ are uniquely identified through the simex procedure, if the extrapolant functions $g(a, \lambda)$ are such that the matrix $\dot{g}(a, \lambda)$ of partial derivatives with respect to the elements of $a$ is bounded and continuous at the 
true parameters $a^{*}$ and has full rank, i.e. $\dot{g}\left(a^{*}, \lambda\right)^{T} \dot{g}\left(a^{*}, \lambda\right)$ is invertible.

The following conditions will be needed in order to establish the asymptotic results.

(A1) With probability one and for some $\tau>0$ we have

$$
\left\|\hat{\gamma}_{\lambda}-\gamma_{\lambda}\right\| \rightarrow 0, \quad\left\|\hat{\beta}_{\lambda}-\beta_{\lambda}\right\| \rightarrow 0 \quad \text { and } \quad \sup _{t \in[0, \tau]}\left|\hat{\Lambda}_{\lambda}(t)-\Lambda_{\lambda}(t)\right| \rightarrow 0
$$

as $n \rightarrow \infty$, i.e. the estimators are strongly consistent. By $\|\cdot\|$ we denote the Euclidean norm.

(A2) For $\mathfrak{m}<\infty$, define

$$
\begin{aligned}
\mathcal{H}_{\mathfrak{m}}=\left\{h=\left(h_{1}, h_{2}, h_{3}\right) \in B V[0, \tau] \times \mathbb{R}^{p} \times \mathbb{R}^{q}:\right. \\
\left.\|h\|_{H}=\left\|h_{1}\right\|_{v}+\left\|h_{2}\right\|+\left\|h_{3}\right\| \leq \mathfrak{m}\right\}
\end{aligned}
$$

where $B V[0, \tau]$ denotes the space of functions of bounded variation on $[0, \tau],\left\|h_{1}\right\|_{v}=\left|h_{1}(0)\right|+V_{0}^{\tau}\left(h_{1}\right)$ and $V_{0}^{\tau}\left(h_{1}\right)$ denotes the total variation of $h_{1}$ over $[0, \tau]$. Uniformly over $h \in \mathcal{H}_{\mathfrak{m}}$ we have

$$
\begin{aligned}
& h_{2}^{T}\left(\hat{\gamma}_{\lambda}-\gamma_{\lambda}\right)+h_{3}^{T}\left(\hat{\beta}_{\lambda}-\beta_{\lambda}\right)+\int_{0}^{\tau} h_{1}(s) \mathrm{d}\left(\hat{\Lambda}_{\lambda}-\Lambda_{\lambda}\right)(s) \\
& =\frac{1}{n} \sum_{i=1}^{n} \Psi_{\lambda}\left(Y_{i}, \Delta_{i}, W_{i, \lambda}, h_{1}, h_{2}, h_{3}\right)+o_{P}\left(n^{-1 / 2}\right)
\end{aligned}
$$

for some function $\Psi_{\lambda}$ such that $\mathbb{E}\left[\Psi_{\lambda}\left(Y, \Delta, W_{\lambda}, h_{1}, h_{2}, h_{3}\right)\right]=0$ and for fixed $\lambda$, the class

$$
\left\{(y, \delta, w) \mapsto \Psi_{\lambda}\left(y, \delta, w, h_{1}, h_{2}, h_{3}\right):\left(h_{1}, h_{2}, h_{3}\right) \in \mathcal{H}_{\mathfrak{m}}\right\}
$$

is uniformly bounded and Donsker.

Theorem 1. Suppose that condition (A1) is satisfied and that $\Lambda^{*}$ is continuous. If the measurement error variance and the true extrapolant functions are known then, with probability one,

$$
\left\|\hat{\gamma}_{\text {simex }}-\gamma^{*}\right\| \rightarrow 0, \quad\left\|\hat{\beta}_{\text {simex }}-\beta^{*}\right\| \rightarrow 0 \quad \text { and } \quad \sup _{t \in[0, \tau]}\left|\hat{\Lambda}_{\text {simex }}(t)-\Lambda^{*}(t)\right| \rightarrow 0
$$

Theorem 2. Suppose that conditions (A1)-(A2) are satisfied and that $\Lambda^{*}$ is continuous. If the measurement error variance and the true extrapolant functions are known, then $n^{1 / 2}\left(\hat{\gamma}_{\text {simex }}-\gamma^{*}\right)$ converges in distribution to $N\left(0, \Sigma_{\gamma}\right)$ and $n^{1 / 2}\left(\hat{\beta}_{\text {simex }}-\beta^{*}\right)$ converges in distribution to $N\left(0, \Sigma_{\beta}\right)$, with $\Sigma_{\gamma}$ and $\Sigma_{\beta}$ as in (11) and (12). Moreover, $n^{1 / 2}\left(\hat{\Lambda}_{\text {simex }}-\Lambda^{*}\right)$ converges weakly in $l^{\infty}([0, \tau])$ to a mean zero Gaussian process $\mathcal{G}$ defined in (13).

The proofs of Theorems 1 and 2 follow the usual arguments for simex estimators. In particular, consistency relies mainly on the consistency of the estimators for each $\lambda$ and consistency of the estimated extrapolant functions. Moreover, the i.i.d. representation in condition (A2) and the expressions in (7) allow us 
to obtain convergence to a Gaussian process for any $\lambda$. Finally, the asymptotic normality of the simex estimators follows by the delta method. Details of the proofs can be found in the Appendix.

The asymptotic variance of the simex estimators can be estimated through a plug-in principle from the formulas (11), (12) and (13) replacing the true parameters $a^{*}$ by their estimators $\hat{a}$ if we are able to estimate the covariance matrix of the naive estimator for each level of added noise $\lambda$. An alternative way to estimate the variance of the simex estimators has been described in Appendix B.4.1 of [11]. The idea is to compute the variance estimator by fitting an extrapolation model and extrapolating to $\lambda=-1$ the matrices $\left\{\Sigma_{\lambda}-\hat{\Sigma}_{\lambda}\right\}_{\lambda \in\left\{\lambda_{1}, \ldots, \lambda_{K}\right\}}$, where $\bar{\Sigma}_{\lambda}=\frac{1}{B} \sum_{b=1}^{B} \operatorname{Var}\left(\hat{\gamma}_{\lambda, b}\right), \operatorname{Var}\left(\hat{\gamma}_{\lambda, b}\right)$ is the estimated covariance matrix of $\hat{\gamma}_{\lambda, b}$ as in the naive estimation method and

$$
\hat{\Sigma}_{\lambda}=\frac{1}{B-1} \sum_{b=1}^{B}\left(\hat{\gamma}_{\lambda, b}-\hat{\gamma}_{\lambda}\right)\left(\hat{\gamma}_{\lambda, b}-\hat{\gamma}_{\lambda}\right)^{T}
$$

is the empirical covariance matrix of $\left\{\hat{\gamma}_{\lambda, b}\right\}_{b=1}^{B}$. Similar expressions hold for $\beta$ and $\Lambda(t)$. However, both these two approaches require estimation of the asymptotic variance for the naive estimation method. The expressions of the asymptotic variances for the estimators of a mixture cure model (without measurement error) are quite complicated $([22,26])$ and in practice estimating them through a plug-in principle (as proposed for example in [22]) is not feasible. For this reason we estimate the variance through a bootstrap procedure as proposed in $[9,26]$.

Remark 1. In practice the true extrapolation function is not known and the mispecification error depends on how good is the approximation of the true extrapolation function by the function we are considering in the simex procedure. For parametric problems, the asymptotic bias resulting from an approximation of the true extrapolation function has been investigated in [13]. To illustrate the idea in our model, we restrict for simplicity to a situation where only one covariate is measured with error, the variance of the measurement error is $\sigma^{2}$ and a quadratic extrapolant is used in the simex procedure. We consider estimation of the first component of $\gamma$ but the idea remains the same for the other parameters. Let $q(\lambda)$ be the true extrapolation function, i.e. $\gamma_{\lambda, 1}=q(\lambda)$ for $\lambda \geq 0$ and $\gamma_{1}^{*}=q(-1)$, where $\gamma_{1}^{*}$ denotes the first component of $\gamma^{*}$. Note that $\gamma_{\lambda, 1}$ depends on $\lambda$ only through $\lambda \sigma^{2}$ because of (6). Hence, by redefining $q$, we can write $\gamma_{\lambda, 1}=q\left(\lambda \sigma^{2}\right)$ and $\gamma_{1}^{*}=q\left(-\sigma^{2}\right)$. Assuming that $q$ is a smooth functional, by a Taylor expansion around $\lambda=-1$, we have

$$
q\left(\lambda \sigma^{2}\right)=a_{1}^{*}+a_{2}^{*} \lambda+a_{3}^{*} \lambda^{2}+\delta_{\lambda},
$$

for some constants $a_{1}^{*}, a_{2}^{*}, a_{3}^{*}$ depending on $\sigma^{2}$ and $\delta_{\lambda}=O\left(\left((\lambda+1) \sigma^{2}\right)^{3}\right)$. In particular, it follows that $\sup _{-1 \leq \lambda \leq C}\left|\delta_{\lambda}\right|=O\left(\sigma^{6}\right)$. For each $\lambda \geq 0$ we have, $\hat{\gamma}_{\lambda, 1} \rightarrow \gamma_{\lambda, 1}$ with probability one as $n \rightarrow \infty$ (see proof of Theorem 1 ). When we fit a quadratic model

$$
\hat{\gamma}_{\lambda, 1}=\gamma_{\lambda, 1}+\epsilon_{\lambda}=a_{1}^{*}+a_{2}^{*} \lambda+a_{3}^{*} \lambda^{2}+\epsilon_{\lambda}+\delta_{\lambda}
$$


using the least-squares method, we obtain estimators $\hat{a}_{1}, \hat{a}_{2}, \hat{a}_{3}$ of $a_{1}^{*}, a_{2}^{*}, a_{3}^{*}$, whose bias $\hat{a}_{i}-a_{i}^{*}$ is a linear function of $\delta_{\lambda}$. Hence, $\left|\hat{a}_{i}-a_{i}^{*}\right|=O\left(\sigma^{6}\right), i=$ $1,2,3$. The resulting extrapolation function is $g(\hat{a}, \lambda)=\hat{a}_{1}+\hat{a}_{2} \lambda+\hat{a}_{3} \lambda^{2}$ and $\hat{\gamma}_{\text {simex }, 1}=g(\hat{a},-1)$. It follows that

$$
\sup _{-1 \leq \lambda \leq C}\left|g(\hat{a}, \lambda)-q\left(\lambda \sigma^{2}\right)\right|=O\left(\sigma^{6}\right)
$$

and in particular $\left|\hat{\gamma}_{\text {simex }, 1}-\gamma_{1}^{*}\right|=O\left(\sigma^{6}\right)$. Hence, in practice even when using an approximation of the true extrapolation function the procedure still works well for small magnitudes of the measurement error variance. The appropriateness of the chosen extrapolation function can also be checked visually through a graphical representation. We investigate the robustness of the method with respect to the extrapolation function through a simulation study in Section 5.4.

\subsection{Example: logistic/Cox mixture cure model}

The logistic/Cox mixture cure model is perhaps the most commonly used one for studying survival data in the presence of a cure fraction. It assumes that the function $\phi(\gamma, x)$ is as in (4), where the first component of $x$ is equal to one and $\gamma_{1}^{*}$ corresponds to the intercept. On the other hand, the distribution of the uncured subjects follows a Cox proportional hazards model as in (5), where $\Lambda^{*}$ is the baseline cumulative hazard, $\beta^{* T} Z$ does not contain an intercept and the matrix $\operatorname{var}(Z)$ is assumed to have full rank for the Cox model to be identifiable. The classical estimator in this setting is the maximum likelihood estimator proposed by [35] and implemented in the $\mathrm{R}$ package smcure. The estimator is computed through the expectation maximization algorithm because of the unobserved cure status and its asymptotic properties are investigated in [22]. Recently, an alternative estimation procedure relying on presmoothing was proposed in [26]. It uses a preliminary nonparametric estimator for the cure probabilities and ignores the Cox model when estimating $\gamma^{*}$. It is shown through simulations that, if the interest is focused on estimation of the parameters of the incidence, this method usually performs better that the maximum likelihood estimator. However, both methods lead to very similar results when estimating the latency. Next we show that these two estimators satisfy our conditions (A1)(A2) and as a result, both procedures can be used in the SIMEX algorithm leading to consistent and square-root convergent estimators.

Theorem 3. Consider the maximum likelihood estimation method proposed by [35]. Assume that conditions 1-4 in [22] are satisfied. Then our conditions (A1)(A2) above hold with $\Psi_{\lambda}\left(y, \delta, w, h_{1}, h_{2}, h_{3}\right)$ as in (18).

Theorem 4. Consider the estimation method proposed by [26] and assume that their assumptions (C1)-(C5), (AC2), (AC5)-(AC7) are satisfied. Then our conditions (A1)-(A2) above hold with $\Psi_{\lambda}\left(y, \delta, w, h_{1}, h_{2}, h_{3}\right)$ as in (20).

For completeness, we list the conditions 1-4 of [22] and the assumptions (C1)(C5), (AC2), (AC5)-(AC7) of [26] in the Appendix. These assumptions are usual 
regularity conditions that guarantee the consistency and the rates of convergence of semiparametric and nonparametric estimators, in this case of the naive estimators (maximum likelihood estimator and the estimator based on presmoothing). More assumptions are needed for the presmoothing approach because it requires nonparametric estimation in the first step. Our condition (A1) in indeed just the consistency of the naive method, while condition (A2) is an intermediate result used to obtain the asymptotic normality of the naive method (in case of semiparametric models). Hence it would be easy to check this condition for any naive method, for which consistency and asymptotic normality have already been obtained.

In order for the mixture cure model to be identifiable, $T_{0}$ should have compact support $\left[0, \tau_{0}\right]$ such that $\inf _{x, z} \mathbb{P}\left(C>\tau_{0} \mid X=x, Z=z\right)>0$. Hence, $\tau$ in our conditions $(A 1)-(A 2)$ is equal to $\tau_{0}$. In practice cure rate models are used when there is a long follow-up beyond the largest observed event time $T_{(m)}$ and the zero-tail constraint is applied, i.e. the censored subjects with follow-up time larger than $T_{(m)}$ are considered cured. For being able to develop the asymptotic theory, in [22] it is assumed that $\inf _{z} \mathbb{P}\left(T_{0}=\tau_{0} \mid Z=z\right)>0$, while [26] argue that this assumption can be avoided thanks to the presmoothing step.

\section{Numerical study}

\subsection{Setup}

In this section we investigate the finite-sample behaviour of the simex method in the logistic/Cox mixture cure model. The two estimation approaches considered in Section 4.2 are used within the simex algorithm and compared with each other in the context of mismeasured covariates. Results for a variety of models and scenarios are presented in the next subsections. We try to cover a wide range of situations and capture the effect of the cure rate, censoring rate, sample size and measurement error variance. Unless stated otherwise, the error distribution is Gaussian and the used extrapolation function is quadratic, which seems to be a good compromise in terms of bias and variance $([13,11,20,4])$. Finally, we also briefly investigate the robustness of the method with respect to the extrapolation function, misspecification of the error distribution and variance. In all the simulation studies, for the simex method, we choose $B=50$, $K=5, \lambda \in\{0,0.5,1,1.5,2\}$ (as these seem to be quite common choices in the literature) and for each setting 500 simulated datasets were used to compute the bias, variance and mean squared error (MSE) of the estimators. We also compare the bias corrected estimators with the naive estimators, which do not take the measurement error into account. The bandwidth for the estimator based on presmoothing is chosen as in [26], i.e. the cross-validation optimal bandwidth for estimation of the conditional distribution $H(t \mid x)$ for $t \leq Y_{(m)}$ truncated from above at 2, where $Y_{(m)}$ is the largest uncensored observation and $x$ is the continuous covariate affecting the incidence (standardized). To reduce computational time, we compute this bandwidth only once for the initial dataset and use the 
same for the data with added noise. We observed that not updating the bandwidth for each $b \in\{1, \ldots, B\}$ and $\lambda \in\{0.5,1,1.5,2\}$ does not have a significant impact on the final results. Moreover, we assume to know the standard deviation of the error, which is usually not the case in practice. In such situations, a preliminary step of variance estimation is required before applying the simex procedure (see for example [6]). Another issue in practice is the correct identification of $X$ and $Z$ since usually one does not know a priori which variables determine the cure status and which affect the survival of the uncured subjects. In our simulation study we assume to know which covariates to include in each part of the model (according to how we generate the data) because the purpose of this study is to investigate the performance of the simex procedure and not problems related to misspecification of $X$ and $Z$. However, in practice one can include all the covariates in both $X$ and $Z$ (if they are not too many) and check their significance for each of the components through the obtained p-values (as we do in Section 6). Another possibility is to first determine the right model, for example based on an Akaike information criterion as suggested in [15], and then apply the simex procedure afterwards to the selected model.

\subsection{One mismeasured covariate}

We start by considering a simplified model in which there is only one covariate of interest, measured with error, affecting both the cure probability and the survival of the uncured subjects.

Model 1. Both incidence and latency depend on one covariate $X$, which is a standard normal random variable. We generate the cure status $B$ as a Bernoulli random variable with success probability $\phi(\gamma, x)=1 /\left(1+\exp \left(-\gamma_{1}-\gamma_{2} x\right)\right)$. The survival times for the uncured observations are generated according to a Weibull proportional hazards model

$$
S_{u}(t \mid x)=\exp \left(-\mu t^{\rho} \exp \left(\beta^{T} x\right)\right)
$$

and are truncated at $\tau_{0}=7$ for $\rho=1.75, \mu=1.5$ and $\beta=1$. The censoring times are independent from $X$ and $T$. They are generated from the exponential distribution with parameter $\lambda_{C}$ and are truncated at $\tau=9$. Various choices of the parameters $\gamma$ and $\lambda_{C}$ with the corresponding cure and censoring rates can be found in Table 1. Here and in what follows, the truncation of the survival times and censoring times is done in such a way that $\tau_{0}<\tau$ and it is unlikely to observe an event time at $\tau_{0}$. This mimics real-life situations in which cure models are adequate. $X$ is measured with error, i.e. instead of $X$ we observe $W=X+U$, where $U \sim N\left(0, v^{2}\right)$.

Results for sample size $n=200(n=400)$ and measurement error variance $v^{2}=0.7^{2}$ are given in Table 2 (Table 7 ). This corresponds to a large error situation since the ratio between the standard deviation of the error and the standard deviation of the covariate is 0.7 . Below we will consider also settings with smaller measurement error. 
TABLE 1

Parameter values and characteristics of each scenario for Model 1.

\begin{tabular}{|c|c|c|c|c|c|c|c|c|}
\hline Setting & $\gamma_{2}$ & Scenario & Cure rate & $\gamma_{1}$ & Cens. rate & $\lambda_{C}$ & Cens. level & Plateau \\
\hline \multirow[t]{4}{*}{1} & \multirow[t]{4}{*}{0.1} & \multirow[t]{2}{*}{1} & \multirow[t]{2}{*}{$20 \%$} & \multirow[t]{2}{*}{1.4} & 1 & 0.09 & $25 \%$ & $14 \%$ \\
\hline & & & & & 2 & 0.3 & $35 \%$ & $7 \%$ \\
\hline & & \multirow[t]{2}{*}{2} & \multirow[t]{2}{*}{$50 \%$} & \multirow[t]{2}{*}{0} & 1 & 0.13 & $55 \%$ & $32 \%$ \\
\hline & & & & & 2 & 0.5 & $65 \%$ & $15 \%$ \\
\hline \multirow[t]{4}{*}{2} & \multirow[t]{4}{*}{0.5} & \multirow[t]{2}{*}{1} & \multirow[t]{2}{*}{$20 \%$} & \multirow[t]{2}{*}{1.4} & 1 & 0.07 & $25 \%$ & $16 \%$ \\
\hline & & & & & 2 & 0.26 & $35 \%$ & $9 \%$ \\
\hline & & \multirow[t]{2}{*}{2} & \multirow[t]{2}{*}{$50 \%$} & \multirow[t]{2}{*}{0} & 1 & 0.15 & $55 \%$ & $31 \%$ \\
\hline & & & & & 2 & 0.6 & $65 \%$ & $14 \%$ \\
\hline \multirow[t]{4}{*}{3} & \multirow[t]{4}{*}{2} & \multirow[t]{2}{*}{1} & \multirow[t]{2}{*}{$20 \%$} & \multirow[t]{2}{*}{2.2} & 1 & 0.1 & $25 \%$ & $15 \%$ \\
\hline & & & & & 2 & 0.33 & $35 \%$ & $9 \%$ \\
\hline & & \multirow[t]{2}{*}{2} & \multirow[t]{2}{*}{$50 \%$} & \multirow[t]{2}{*}{0} & 1 & 0.2 & $55 \%$ & $33 \%$ \\
\hline & & & & & 2 & 0.7 & $65 \%$ & $16 \%$ \\
\hline
\end{tabular}

First of all, we observe that in the presence of measurement error there is usually no advantage of using the presmoothing approach instead of maximum likelihood estimation. In particular, when the bias induced by the measurement error is large, it seems that the estimator based on presmoothing is more affected for both the naive and the simex method. Moreover, most of the time the bias is observed only for the coefficients that correspond to the variables measured with error. As expected, in all cases, the simex algorithm reduces this bias at the price of a larger variance. In terms of mean squared error, it is better to use the naive approach for coefficients that are small in absolute value (the case of $\gamma_{2}$ in setting 1), while the simex method is preferred when the absolute value of the coefficient is large (i.e. the covariate has a greater effect on the cure/survival). In this setting, for $n=200, \gamma_{2}=0.5$ seems to be a borderline case, meaning that the simex method performs better when the censoring rate is low, while the naive method has smaller MSE when the censoring rate is high. In addition, results show that when the coefficient of a mismeasured covariate is large, there might be induced bias even for the intercept, which is also corrected by the simex algorithm. As the sample size increases, the bias created by the measurement error increases but the variance decreases for both naive and simex estimators. Furthermore, the advantage of using simex instead of ignoring the bias becomes more significant. At the same time, the threshold absolute value of a coefficient for which bias correction leads to better MSE decreases (simex is preferred for $\gamma_{2}=0.5$ in setting 2 , which was a borderline case for $n=200$ ).

\subsection{More realistic scenarios}

Through the following four models we try to cover more realistic situations and investigate the effect of the measurement error on the naive and bias corrected estimators.

Model 2. Both incidence and latency depend on two independent covariates: $X_{1}$ has a uniform distribution on the interval $[-1,1]$ and $X_{2}$ is a Bernoulli random variable with success probability 0.5 . We generate the cure status $B$ as 
TABLE 2

Bias, variance and MSE of $\hat{\gamma}$ and $\hat{\beta}$ for the naive and simex method based on the maximum likelihood (1) or the presmoothing (2) approach for Model 1 ( $n=200)$. The first column gives the setting/scenario/cens. level. All numbers were multiplied by 100.

\begin{tabular}{|c|c|c|c|c|c|c|c|c|c|c|c|c|c|}
\hline & \multirow[b]{2}{*}{ Par. } & \multicolumn{3}{|c|}{ naive - 1} & \multicolumn{3}{|c|}{ naive - 2} & \multicolumn{3}{|c|}{ simex - 1} & \multicolumn{3}{|c|}{ simex -2} \\
\hline & & Bias & Var. & MSE & Bias & Var. & MSE & Bias & Var. & MSE & Bias & Var. & MSE \\
\hline \multirow[t]{3}{*}{$\overline{1 / 1 / 1}$} & $\gamma_{1}$ & 2.4 & 3.8 & 3.8 & 1.5 & 3.8 & 3.8 & 2.4 & 3.8 & 3.9 & 1.4 & 4.0 & 4.0 \\
\hline & $\gamma_{2}$ & -3.5 & 2.6 & 2.7 & -2.7 & 2.5 & 2.5 & -1.2 & 4.8 & 4.8 & -0.2 & 5.1 & 5.1 \\
\hline & $\beta$ & -43.3 & 0.7 & 19.4 & -43.3 & 0.7 & 19.4 & -18.8 & 1.9 & 5.5 & -18.9 & 1.9 & 5.5 \\
\hline \multirow[t]{3}{*}{$1 / 1 / 2$} & $\gamma_{1}$ & 3.9 & 5.5 & 5.6 & 0.5 & 5.1 & 5.2 & 3.6 & 5.6 & 5.7 & -0.3 & 6.2 & 6.2 \\
\hline & $\gamma_{2}$ & -3.0 & 4.0 & 4.1 & 0.3 & 3.8 & 3.9 & -0.2 & 7.3 & 7.3 & 4.5 & 8.6 & 8.8 \\
\hline & $\beta$ & -42.4 & 0.9 & 18.9 & 42.8 & 0.9 & 19.2 & -18.2 & 2.4 & 5.7 & -18.6 & 2.4 & 5.9 \\
\hline \multirow[t]{3}{*}{$1 / 2 / 1$} & $\gamma_{1}$ & 0.7 & 2.4 & 2.4 & 0.2 & 2.4 & 2.4 & 0.7 & 2.4 & 2.4 & 0.2 & 2.5 & 2.5 \\
\hline & $\gamma_{2}$ & -4.0 & 1.6 & 1.8 & -3.6 & 1.4 & 1.5 & -1.9 & 3.0 & 3.0 & -1.3 & 2.7 & 2.7 \\
\hline & $\beta$ & -42.5 & 1.2 & 19.3 & -42.6 & 1.2 & 19.3 & -18.1 & 3.3 & 6.6 & -18.2 & 3.3 & 6.6 \\
\hline \multirow[t]{3}{*}{$1 / 2 / 2$} & $\gamma_{1}$ & 1.6 & 4.2 & 4.2 & -0.3 & 4.0 & 4.0 & 1.3 & 4.3 & 4.3 & -0.2 & 4.7 & 4.7 \\
\hline & $\gamma_{2}$ & -3.0 & 2.8 & 2.9 & -2.1 & 2.4 & 2.5 & -0.4 & 5.0 & 5.0 & 0.4 & 5.2 & 5.2 \\
\hline & $\beta$ & -41.9 & 1.9 & 19.5 & -42.3 & 1.8 & 19.8 & -18.0 & 4.9 & 8.1 & -18.4 & 4.7 & 8.1 \\
\hline \multirow[t]{3}{*}{$2 / 1 / 1$} & $\gamma_{1}$ & -0.3 & 3.7 & 3.7 & -1.0 & 3.6 & 3.7 & 1.4 & 3.9 & 4.0 & 0.4 & 4.1 & 4.1 \\
\hline & $\gamma_{2}$ & -15.9 & 2.3 & 4.9 & -16.6 & 2.2 & 5.0 & -3.9 & 4.5 & 4.7 & -5.1 & 4.7 & 5.0 \\
\hline & $\beta$ & -44.2 & 0.7 & 20.2 & 44.2 & 0.7 & 20.2 & -19.7 & 2.0 & 5.8 & -19.7 & 2.0 & 5.8 \\
\hline \multirow[t]{3}{*}{$2 / 1 / 2$} & $\gamma_{1}$ & 2.1 & 4.8 & 4.8 & -0.5 & 4.7 & 4.7 & 3.8 & 5.2 & 5.3 & 0.8 & 5.8 & 5.8 \\
\hline & $\gamma_{2}$ & -16.0 & 3.6 & 6.2 & -14.8 & 3.4 & 5.6 & -3.6 & 7.0 & 7.1 & -1.5 & 7.9 & 8.0 \\
\hline & $\beta$ & -43.1 & 0.9 & 19.4 & -43.3 & 0.9 & 19.6 & -18.6 & 2.4 & 5.9 & -18.9 & 2.4 & 5.9 \\
\hline \multirow[t]{3}{*}{$2 / 2 / 1$} & $\gamma_{1}$ & 0.8 & 2.3 & 2.3 & 0.3 & 2.3 & 2.3 & 0.8 & 2.4 & 2.4 & 0.2 & 2.5 & 2.5 \\
\hline & $\gamma_{2}$ & -16.7 & 1.8 & 4.6 & -17.9 & 1.7 & 4.9 & -4.8 & 3.5 & 3.7 & -6.3 & 3.5 & 3.9 \\
\hline & $\beta$ & -43.6 & 1.3 & 20.3 & -43.6 & 1.3 & 20.3 & -19.1 & 3.5 & 7.2 & -19.1 & 3.5 & 7.2 \\
\hline \multirow[t]{3}{*}{$2 / 2 / 2$} & $\gamma_{1}$ & 0.6 & 4.7 & 4.7 & -1.7 & 4.3 & 4.3 & 0.1 & 4.8 & 4.8 & -2.2 & 4.9 & 4.9 \\
\hline & $\gamma_{2}$ & -15.0 & 3.4 & 5 . & -16.3 & 3.1 & 5 . & -2.2 & 6.4 & 6. & -4.0 & 6.5 & 6.7 \\
\hline & $\beta$ & -43.1 & 2.0 & 2 & -43.2 & & 20.5 & -19.3 & 5.0 & 8 & -19.3 & 4.8 & 8.5 \\
\hline \multirow{3}{*}{$3 / 1 / 1$} & $\gamma_{1}$ & -33.6 & 7.1 & 18.4 & -36.6 & 6.8 & 20.1 & -12.1 & 13.3 & 14.8 & -16.5 & 12.9 & 15.6 \\
\hline & $\gamma_{2}$ & -84.6 & 6.0 & 77.5 & -88.6 & 5.4 & 84.0 & -34.0 & 17.0 & 28.6 & -40.3 & 16.1 & 32.3 \\
\hline & $\beta$ & -48.0 & 0.8 & 23.9 & -48.0 & 0.8 & 23.8 & -23.1 & 2.3 & 7.6 & -23.0 & 2.3 & 7.6 \\
\hline \multirow[t]{3}{*}{$3 / 1 / 2$} & $\gamma_{1}$ & -31.0 & 10.6 & 20.3 & -37.2 & 9.8 & 23.7 & -9.0 & 19.1 & 19.9 & -17.4 & 19.5 & 22.6 \\
\hline & $\gamma_{2}$ & -83.7 & 8.5 & 78.5 & -89.2 & 7.4 & 86.9 & -32.0 & 23.6 & 33.8 & -40.6 & 22.4 & 38.9 \\
\hline & $\beta$ & -46.8 & 1.0 & 22.9 & -46.7 & 1.0 & 22.8 & -21.9 & 2.7 & 7.4 & -21.8 & 2.6 & 7.4 \\
\hline \multirow[t]{3}{*}{$3 / 2 / 1$} & $\gamma_{1}$ & 0.9 & 2.9 & 2.9 & 0.4 & 2.8 & 2.8 & 1.0 & 3.8 & 3.8 & 0.7 & 3.9 & 3.9 \\
\hline & $\gamma_{2}$ & -88.8 & 4.0 & 82.9 & -93.6 & 3.8 & 91.4 & -38.6 & 11.0 & 25.9 & -46.1 & 11.0 & 32.2 \\
\hline & $\beta$ & -51.1 & 1.6 & 27.7 & -50.9 & 1.6 & 27.5 & -26.5 & 4.7 & 11.7 & -26.2 & 4.7 & 11.5 \\
\hline \multirow[t]{3}{*}{$3 / 2 / 2$} & $\gamma_{1}$ & 1.8 & 5.2 & 5.3 & -0.8 & 4.9 & 4.9 & 1.8 & 6.9 & 6.9 & -1.0 & 6.9 & 6.9 \\
\hline & $\gamma_{2}$ & -87.6 & 6.7 & 83.6 & -95.4 & 5.8 & 96.9 & -37.0 & 19.3 & 33.0 & -48.9 & 17.6 & 41.5 \\
\hline & $\beta$ & -49.5 & 2.2 & 26.7 & -48.9 & 2.1 & 26.0 & -24.4 & 6.1 & 12.1 & -23.7 & 5.7 & 11.3 \\
\hline
\end{tabular}

a Bernoulli random variable with success probability

$$
\phi(\gamma, x)=1 /\left(1+\exp \left(-\gamma_{1}-\gamma_{2} x_{1}-\gamma_{3} x_{2}\right)\right) \text {. }
$$

The survival times for the uncured observations are generated according to a Weibull proportional hazards model

$$
S_{u}(t \mid x)=\exp \left(-\mu t^{\rho} \exp \left(\beta_{1} x_{1}+\beta_{2} x_{2}\right)\right),
$$

and are truncated at $\tau_{0}$ for $\rho=1.75$ and $\mu=1.5$. The censoring times are independent from $(X, T)$. They are generated from the exponential distribution with parameter $\lambda_{C}$ and are truncated at $\tau$. Instead of $X_{1}$ we observe $W=$ $X_{1}+U$, where $U \sim N\left(0, v^{2}\right)$. We consider $v \in\{0.2,0.4\}$ corresponding to small and large error settings respectively.

Model 3. For the incidence we consider two independent covariates: $X_{1}$ has a uniform distribution on the interval $[-1,1]$ and $X_{2}$ is a Bernoulli random 
variable with success probability 0.5 . The latency also depends on two covariates: $Z_{1}=X_{1}$ and $Z_{2}$ is independent of the previous ones and normally distributed with mean zero and standard deviation 0.3 . We generate the cure status $B$ as a Bernoulli random variable with success probability

$$
\phi(\gamma, x)=1 /\left(1+\exp \left(-\gamma_{1}-\gamma_{2} x_{1}-\gamma_{3} x_{2}\right)\right) .
$$

The survival times for the uncured observations are generated according to a Weibull proportional hazards model

$$
S_{u}(t \mid z)=\exp \left(-\mu t^{\rho} \exp \left(\beta_{1} z_{1}+\beta_{2} z_{2}\right)\right),
$$

and are truncated at $\tau_{0}$ for $\rho=1.75$ and $\mu=1.5$. The censoring times are independent from $(T, X, Z)$. They are generated from the exponential distribution with parameter $\lambda_{C}$ and are truncated at $\tau$. The mismeasured covariate is $Z_{2}$, i.e. we only observe $W=Z_{2}+U$, where $U \sim N\left(0, v^{2}\right)$ and $v \in\{0.1,0.2\}$ corresponding to small and large error settings respectively.

Model 4. The incidence depends on one covariate $X$ which is a standard normal random variable. The latency depends on two covariates: $Z_{1}=X$ and $Z_{2}$ is independent of $X$ and uniformly distributed on $[-1,1]$. We generate the cure status $B$ as a Bernoulli random variable with success probability $\phi(\gamma, x)=$ $1 /\left(1+\exp \left(-\gamma_{1}-\gamma_{2} x\right)\right)$. The survival times for the uncured observations are generated according to a Weibull proportional hazards model

$$
S_{u}(t \mid z)=\exp \left(-\mu t^{\rho} \exp \left(\beta_{1} z_{1}+\beta_{2} z_{2}\right)\right),
$$

and are truncated at $\tau_{0}$ for $\rho=1.75$ and $\mu=1.5$. The censoring times are independent of the vector $(X, Z, T)$. They are generated from the exponential distribution with parameter $\lambda_{C}$ and are truncated at $\tau$. Instead of $X$ and $Z_{2}$ we observe $W_{1}=X+U_{1}$ and $W_{2}=Z_{2}+U_{2}$, where the error terms $U_{1} \sim N\left(0, v_{1}^{2}\right)$ and $U_{2} \sim N\left(0, v_{2}^{2}\right)$ are independent. We consider $\left(v_{1}, v_{2}\right)=(0.35,0.2)$ and $\left(v_{1}, v_{2}\right)=(0.7,0.4)$ corresponding to small and large error settings respectively.

Model 5. The incidence depends on one covariate $X$ which is a standard normal random variable. The latency depends on two correlated covariates: $Z_{1}=X$ and $Z_{2}=-X+N$, where $N$ is a normal random variable with mean zero and standard deviation 0.5 independent of $X$. We generate the cure status $B$ as a Bernoulli random variable with success probability

$$
\phi(\gamma, x)=1 /\left(1+\exp \left(-\gamma_{1}-\gamma_{2} x\right)\right) .
$$

The survival times for the uncured observations are generated according to the Weibull proportional hazards model in (9) and are truncated at $\tau_{0}$ for $\rho=1.75$ and $\mu=1.5$. The censoring times are independent of the vector $(X, Z, T)$. They are generated from the exponential distribution with parameter $\lambda_{C}$ and are truncated at $\tau$. The covariate $Z_{2}$ is measured with error, i.e. instead of $Z_{2}$ we observe $W=Z_{2}+U$, where $U \sim N\left(0, v^{2}\right)$ is independent of the previous variables. We consider $v=0.39$ and $v=0.78$ corresponding to small and large error settings respectively. 
TABLE 3

Parameter values and model characteristics for each scenario in Models 2-5.

\begin{tabular}{ccccccccc}
\hline Model & Scenario & $\gamma^{*}$ & $\beta^{*}$ & $\lambda_{C}$ & $\left(\tau_{0}, \tau\right)$ & $\begin{array}{c}\text { Cure } \\
\text { rate }\end{array}$ & $\begin{array}{c}\text { Cens. } \\
\text { rate }\end{array}$ & Plateau \\
\hline \multirow{2}{*}{2} & 1 & $(1.3,1,0.4)$ & $(0.8,0.3)$ & 0.33 & $(4,6)$ & $20 \%$ & $35 \%$ & $9 \%$ \\
& 2 & $(1.1,1.3,-0.3)$ & $(2,-0.8)$ & 0.08 & $(10,12)$ & $30 \%$ & $35 \%$ & $19 \%$ \\
& 3 & $(-0.5,1.5,1)$ & $(0.8,0.3)$ & 0.4 & $(4,6)$ & $50 \%$ & $60 \%$ & $22 \%$ \\
3 & 1 & $(1.3,1,0.4)$ & $(1.5,0.5)$ & 0.3 & $(6,8)$ & $20 \%$ & $35 \%$ & $7 \%$ \\
& 2 & $(1.1,1.3,-0.3)$ & $(1,-1)$ & 0.1 & $(6,8)$ & $30 \%$ & $35 \%$ & $22 \%$ \\
& 3 & $(-0.5,1.5,1)$ & $(0.5,1.5)$ & 0.3 & $(6,8)$ & $50 \%$ & $60 \%$ & $24 \%$ \\
& 1 & $(1.4,0.5)$ & $(0.5,0.1)$ & 0.3 & $(5,7)$ & $20 \%$ & $35 \%$ & $9 \%$ \\
& 2 & $(1.4,2)$ & $(0.1,0.5)$ & 0.12 & $(5,7)$ & $30 \%$ & $35 \%$ & $22 \%$ \\
& 3 & $(0 .-2)$ & $(-1.5,0.5)$ & 0.5 & $(5,7)$ & $50 \%$ & $60 \%$ & $14 \%$ \\
& 1 & $(1.4,0.5)$ & $(0.5,0.1)$ & 0.3 & $(4,6)$ & $20 \%$ & $35 \%$ & $10 \%$ \\
5 & 2 & $(1.4,2)$ & $(0.1,-0.5)$ & 0.13 & $(4,6)$ & $30 \%$ & $35 \%$ & $21 \%$ \\
& 3 & $(0,2)$ & $(1,-1)$ & 0.5 & $(6,8)$ & $50 \%$ & $60 \%$ & $12 \%$ \\
\hline
\end{tabular}

For the four models, various choices of the parameters $\gamma, \beta, \lambda_{C}$ and $\left(\tau_{0}, \tau\right)$ are considered, in such a way that we obtain three scenarios for the cure rate $(20 \%, 30 \%$ and $50 \%)$ and different levels of censoring (see Table 3). The sample size is fixed at $n=200$, while the variance of the measurement error is chosen as described in each model, corresponding to a ratio between the standard deviation of the error and the standard deviation of the covariate equal to 0.35 and 0.7. Results for some of the settings are given in Tables 4 and the rest in Table 8 .

Once more we observe that the maximum likelihood estimator and the estimator based on presmoothing give comparable results for both the naive and the simex method. As expected, the measurement error mainly affects the estimators of the coefficients corresponding to the mismeasured covariates. However, the measurement error induces bias also on variables correlated to the mismeasured covariate within the same component. For example in Model 5, the measurement error of $Z_{2}$ leads to biased estimators for $\beta_{1}$ and $\beta_{2}$, but does not affect the estimation of $\gamma_{2}$ even though $Z_{1}=X$. In all settings, the simex method corrects for the bias due to the measurement error. Nevertheless, in terms of mean squared error, the naive approach is still preferred when the measurement error is small and the absolute value of the coefficient corresponding to the standardized covariate is small (the covariate has a weak effect on cure or survival). On the contrary, a strong effect (large coefficient) and a large measurement error favour the use of the simex method.

\subsection{Robustness of the method}

Here we investigate the robustness of the simex approach with respect to the choice of the extrapolation function, misspecification of the error distribution and of the error standard deviation. We focus on Model 2, where the mismeasured covariate is $X_{1}=Z_{1}$ affecting both the cure probability and the survival. The sample size is $n=200$ and the error standard deviation is $v=0.2$ or $v=0.4$. 
TABLE 4

Bias, variance and MSE of $\hat{\gamma}$ and $\hat{\beta}$ for the naive and simex method based on the maximum likelihood (1) or the presmoothing (2) approach in Models 2-5 $(n=200)$. The first column gives the model, scenario and the standard deviation of the measurement error. All numbers were multiplied by 100.

\begin{tabular}{|c|c|c|c|c|c|c|c|c|c|c|c|c|c|}
\hline \multirow[b]{2}{*}{ Mod./Scen./v } & \multirow[b]{2}{*}{ Par. } & \multicolumn{3}{|c|}{ naive - 1} & \multicolumn{3}{|c|}{ naive -2} & \multicolumn{3}{|c|}{ simex - 1} & \multicolumn{3}{|c|}{ simex - 2} \\
\hline & & Bias & Var. & MSE & Bias & Var. & MSE & Bias & Var. & MSE & Bias & Var. & MSE \\
\hline \multirow[t]{5}{*}{$2 / 1 / 0.2$} & $\gamma_{1}$ & 4.8 & 12.0 & 12.2 & 2.7 & 11.6 & 11.6 & 5.9 & 12.6 & 13.0 & 4.5 & 13.0 & 13.2 \\
\hline & $\gamma_{2}$ & -6.3 & 18.3 & 18.7 & -11.3 & 16.2 & 17.5 & 4.7 & 24.6 & 24.8 & -1.0 & 23.9 & 23.9 \\
\hline & $\gamma_{3}$ & -1.1 & 25.0 & 25.0 & -1.7 & 25.3 & 25.3 & -0.7 & 25.4 & 25.4 & -2.2 & 29.4 & 29. \\
\hline & $\beta_{1}$ & -8.3 & 2.9 & 3.6 & -8.1 & 2.9 & 3.6 & 1.0 & 4.0 & 4.0 & 1.2 & 4.0 & \\
\hline & $\beta_{2}$ & -0.2 & 4.2 & 4.2 & -0.2 & 4.1 & 4.1 & 0.4 & 4.3 & 4.3 & 0.5 & 4.3 & \\
\hline \multirow[t]{5}{*}{$2 / 1 / 0.4$} & $\gamma_{1}$ & 2.3 & 11.8 & 11.8 & 0.1 & 11.7 & 11.7 & 4.4 & 13.1 & 13.3 & 2.6 & 14.8 & 14.9 \\
\hline & $\gamma_{2}$ & -30.3 & 13.4 & 22.5 & -34.4 & 12.1 & 24.0 & -6.0 & 27.0 & 27.3 & -11.4 & 28.5 & 29. \\
\hline & $\gamma_{3}$ & -1.3 & 25.0 & 25.0 & -2.2 & 24.7 & 24.8 & -0.6 & 26.3 & 26.3 & -1.7 & 30.1 & 50. \\
\hline & $\beta_{1}$ & -27.2 & 2.1 & 9.5 & -27.1 & 2.0 & 9.4 & -7.9 & 4.3 & 5.0 & -7.7 & 4.3 & \\
\hline & $\beta_{2}$ & -1.3 & 4.2 & 4.2 & -1.3 & 4.2 & 4.2 & 0.0 & 4.6 & 4.6 & 0.0 & 4.6 & \\
\hline \multirow[t]{5}{*}{$2 / 2 / 0.2$} & $\gamma_{1}$ & 2.5 & 6.8 & 6.9 & 1.9 & 6.8 & 6.8 & 4.0 & 7.1 & 7.2 & 3.7 & 7.5 & \\
\hline & $\gamma_{2}$ & -11.7 & 10.2 & 11.6 & -13.9 & 9.8 & 11.8 & 2.0 & 13.4 & 13.4 & -0.3 & 13.9 & 14 \\
\hline & & -2.8 & 12.7 & 12.8 & -2.9 & 12.7 & 12.8 & -3.0 & 13.0 & 13.1 & -3.3 & 13.9 & 14 \\
\hline & $\beta$ & -33.3 & 4.1 & 15.2 & -33.3 & 4.1 & 15.2 & -1.4 & 7.9 & 7.9 & -1.4 & 7.9 & \\
\hline & $\beta_{2}$ & 6.1 & 4.3 & 4.7 & 6.1 & 4.3 & 4.7 & 0.0 & 5.3 & 5.3 & 0.0 & 5.3 & \\
\hline \multirow[t]{5}{*}{$2 / 2 / 0.4$} & $\gamma_{1}$ & -0.7 & 6.5 & 6.5 & -1.4 & 6.6 & 6.6 & 2.2 & 7.2 & 7.2 & 1.7 & 8.0 & \\
\hline & $\gamma_{2}$ & -41.8 & 7.4 & 24.9 & -42.9 & 7.3 & 25.7 & -10.7 & 15.0 & 16.2 & -11.5 & 16.4 & 17. \\
\hline & $\gamma_{3}$ & -1.7 & 12.4 & 12.4 & -2.1 & 12.4 & 12.4 & -2.3 & 13.2 & 13.3 & -3.0 & 14.5 & 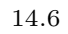 \\
\hline & $\beta_{1}$ & -89.9 & 2.5 & 83.3 & -89.9 & 2.5 & 83.3 & -41.1 & 7.3 & 24.2 & -41.1 & 7.3 & 24 \\
\hline & $\beta_{2}$ & 16.8 & 4.5 & 7.4 & 16.9 & 4.5 & 7.4 & 8.1 & 6.5 & 7.1 & 8.1 & 6.5 & \\
\hline \multirow[t]{5}{*}{$3 / 3 / 0.1$} & $\gamma_{1}$ & -1.8 & 7.4 & 7.5 & -1.5 & 7.5 & 7.6 & -1.8 & 7.4 & 7.5 & -1.5 & 7.5 & \\
\hline & $\gamma$ & 5.0 & 13.4 & 13.6 & -2.8 & 13.6 & 13.7 & 4.9 & 13.4 & 13.6 & -2.8 & 13.6 & 10 \\
\hline & $\gamma_{3}$ & 3.9 & 15.2 & 15.4 & 2.0 & 15.9 & 16.0 & 3.9 & 15.2 & 15.4 & 2.0 & 15.9 & 16 \\
\hline & $\beta_{1}$ & -0.3 & 6.4 & 6.4 & 0.4 & 6.3 & 6.3 & 0.3 & 6.5 & 6.5 & 1.0 & 6.4 & \\
\hline & $\beta_{2}$ & -18.7 & 17.1 & 20.5 & -19.1 & 17.0 & 20.7 & -3.2 & 22.7 & 22.8 & -3.6 & 22.7 & 22 . \\
\hline \multirow[t]{5}{*}{$3 / 3 / 0.2$} & $\gamma_{1}$ & -1.8 & 7.4 & 7.5 & -1.5 & 7.5 & 7.6 & -1.8 & 7.5 & 7.5 & -1.5 & 7.5 & \\
\hline & $\gamma_{2}$ & 5.0 & 13.5 & 13.7 & -2.8 & 13.6 & 13.7 & 5.0 & 13.5 & 13.7 & -2.8 & 13.6 & 13. \\
\hline & & 3.9 & 15.2 & 15.3 & 2.0 & 15.9 & 15.9 & 4.0 & 15.2 & & 2.0 & 15.9 & 15. \\
\hline & $\beta_{1}$ & -1.6 & 6.4 & 6.4 & -0.9 & 6.3 & 6.3 & -0.4 & 6.8 & 6.8 & 0.3 & 6.7 & \\
\hline & $\beta_{2}$ & -51.6 & 13.1 & 39.7 & -52.0 & 13.0 & 40.0 & -17.8 & 26.9 & 30.0 & -18.2 & 26.9 & 30. \\
\hline $4 / 3$ & $\gamma_{1}$ & 0.7 & 6.1 & 6.1 & -1.2 & 5.9 & 5.9 & 0.3 & 7.1 & 7.1 & -1.4 & 7.4 & \\
\hline$v_{1}=0.35$ & $\gamma_{2}$ & 29.4 & 9.9 & 18.6 & 37.7 & 10.5 & 24.8 & -3.7 & 18.3 & 18.4 & 8.2 & 20.8 & 21. \\
\hline \multirow[t]{2}{*}{$v_{2}=0.2$} & $\beta_{1}$ & 33.6 & 3.9 & 15.2 & 33.3 & 4.0 & 15.0 & 3.7 & 8.2 & 8.3 & 3.2 & 8.2 & \\
\hline & $\beta_{2}$ & -9.0 & 4.8 & 5.6 & -9.3 & 4.7 & 6 & -0.8 & 7.6 & 7.6 & -1.0 & 7.5 & \\
\hline $4 / 3$ & $\gamma_{1}$ & 1.2 & 5.2 & 5.2 & -0.9 & 4.9 & .0 & 0.9 & 6.9 & 6.9 & -1.0 & 6.9 & \\
\hline$v_{1}=0$. & 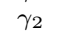 & 89.1 & 4.9 & 84.3 & 93.9 & 5.0 & 93.1 & 38.8 & 13.8 & 28.9 & 46.6 & 14.7 & 0 \\
\hline \multirow[t]{2}{*}{$v_{2}=0.4$} & $\beta_{1}$ & 82.2 & 2.6 & 70.2 & 82.0 & 2.5 & 69.8 & 46.2 & 7.8 & 9.2 & 45.9 & 7.6 & \\
\hline & $\beta_{0}$ & -23.1 & 3. & 9.0 & -23.3 & 3.6 & 0 & 11.2 & 8.7 & 10.0 & -11.5 & 8. & \\
\hline $5 / 1 / 0.39$ & $\gamma_{1}$ & 3.2 & 4.9 & 5.0 & 1.2 & 4.7 & 7 & 3.2 & 5.0 & 5.1 & 1.2 & 4. & \\
\hline & $\gamma_{2}$ & 2.9 & 5. & 5.6 & -0.5 & 5.3 & & & 5. & & -0.5 & 5 & \\
\hline & p & -3.7 & 3.3 & 3.4 & -3.5 & 3.2 & 3.4 & -1.5 & $5 .^{\prime}$ & 5.7 & -1.3 & 5. & \\
\hline & $\beta_{2}$ & -4.3 & 2.4 & 2.5 & -4.3 & 2.3 & 25 & -2.0 & 4.7 & 4.8 & -2.1 & 4.7 & \\
\hline $5 / 1 / 0.78$ & $\gamma_{1}$ & 3.1 & 4.9 & 5.0 & 1.2 & 4.7 & & 3.2 & 5. & 5 & 1.2 & 4. & \\
\hline & 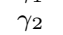 & 2.9 & 5.5 & 5.6 & -0.5 & 5. & & 2.9 & 5. & & -0.5 & 5. & \\
\hline & $\beta_{1}$ & -6.7 & 2.1 & 2.5 & -6.5 & 2.0 & 2.5 & -5.0 & 4. & 4.3 & -4.8 & 4. & \\
\hline & $\beta_{2}$ & -7.2 & 1.1 & 1.6 & -7.3 & 1.1 & & -5.4 & 3. & 3 & -5.5 & 3.1 & \\
\hline $5 / 2 / 0.39$ & $\gamma_{1}$ & 6.1 & 7.9 & 8.3 & 2.2 & 7.1 & 7.2 & 6.1 & 7.9 & 0.5 & 2.2 & 7.1 & \\
\hline & 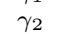 & 8.6 & 14.5 & 15.2 & -1.8 & 12.5 & 12.6 & 8.6 & 14.5 & 15.2 & -1.8 & 12.5 & 12 \\
\hline & 12 & 18.4 & 3.2 & 6.6 & 18.5 & 3.2 & 6. & 6.6 & 5.2 & & 6.7 & 5.2 & \\
\hline & $\beta_{2}$ & 18.6 & 2.2 & 5. & 18.6 & 2.2 & & 6.1 & 4.5 & 4. & 6.2 & 4.5 & \\
\hline $5 / 2 / 0.78$ & $\gamma_{1}$ & 6.2 & 8.0 & 8.3 & 2.2 & 7.1 & 7.2 & 6.1 & 8.0 & 8.4 & 2.2 & 71. & \\
\hline & . & 8.7 & 14.5 & & -1.8 & 12.5 & 12.6 & 8.6 & 14.6 & 15.3 & -1.8 & 12.5 & \\
\hline & 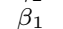 & 34.3 & 2.3 & 14 & 34.4 & 2.3 & 14. & 5.3 & 3.9 & 10.3 & 25.4 & 3.8 & 10 . \\
\hline & $\beta_{2}$ & 35.5 & 1.0 & 13.6 & 35.5 & 1.0 & 13.6 & 26.0 & 2.8 & 9.6 & 26.0 & 2.8 & \\
\hline
\end{tabular}

In addition to the quadratic extrapolant used in Table 4, we consider also a linear and a cubic extrapolant. Results in Table 9 show that, as the order of the 
extrapolation function increases, the difference between the maximum likelihood estimators and the estimators based on presmoothing becomes more significant. In particular, it favours the first method over the latter one mainly due to a smaller variance. As expected, the choice of the extrapolation function has stronger effect on the coefficients corresponding to the mismeasured covariates and when the error is large. For $v=0.4$, the bias decreases as the extrapolation order increases while there is no clear conclusion when $v$ is small. In terms of mean squared error, linear extrapolation is preferred when the measurement error variance is low or more in general in situations where the naive method would do better than the simex approach. In cases where simex outperforms the naive estimators, the quadratic extrapolant seems to be the best choice.

To understand what happens if the error distribution is misspecified we generate the measurement error from three other distributions: a uniform distribution $U \sim \operatorname{Unif}(-a, a)$, a Student-t distribution with $k$ degrees of freedom $a^{-1} U \sim t_{k}$ and a chi-squared distribution with $k$ degrees of freedom $a^{-1} U+k \sim \chi_{k}^{2}$. The constant $a$ is chosen in such a way that the standard deviation of $U$ is $v=0.2$ or $v=0.4$. In all three cases we still use the Gaussian distribution in the simex procedure. Results are given in Table 10. We observe that, when the true distribution is uniform or Student-t, the method still behaves quite well and there is little impact on the estimators. However, when the true distribution is not symmetric $\left(\chi^{2}\right)$ there is a significant increase in mean squared error, in particular for large $v$.

Finally we investigate the effect of error variance misspecification. We simulate the error from a normal distribution with standard deviation $v=0.2$ and $v=0.4$ but in the estimation process the variance is misspecified $v_{E} \in$ $\{v-0.1, v+0.1\}$. Results reported in Table 11 show that the misspecification affects estimation of all the parameters but the difference is larger for those that correspond to the mismeasured covariates. As expected, increasing the specified variance $v_{E}^{2}$ leads to an increased variance of the simex estimators. For small $v$, the lowest bias is obtained when $v$ is correctly specified while for large $v$, the bias decreases as the specified variance increases. In terms of mean squared error, in situations where simex performs worse than the naive approach underspecifying the variance works better. On the other hand, when simex outperforms the naive estimators, overspecifying the error variance is preferred over underspecification.

\section{Application: prostate cancer study}

In this section we illustrate the practical use of the proposed simex procedure for a medical dataset concerning patients with prostate cancer. According to the American Cancer Society, prostate cancer is the second most common cancer among American men (after skin cancer) and it is estimated that about 1 man in 9 is diagnosed with prostate cancer during his lifetime. Even though most men diagnosed with prostate cancer do not die from it, it can sometimes be a serious disease. The 5 -year survival rate based on the stage of the cancer at diagnoses is almost $100 \%$ for localized or regional stage and drops to $31 \%$ for distant stage. 
Among other factors, the prostate-specific antigen (PSA) blood level is a good indicator of the presence of the cancer and is used as a tool to both diagnose and monitor the development of the disease. In most cases, elevated PSA levels indicate a poor prostate cancer prognosis. Even though most studies do not take it in consideration, the PSA measurements are not error-free because of the inaccuracy of the measuring technique and own fluctuations of the PSA levels. Here we try to analyse the effect of PSA on cure probability and survival while accounting for measurement error. Our purpose is to check how sensitive the results are to the measurement error.

We obtain the data from the Surveillance, Epidemiology and End Results (SEER) database, which is a collection of cancer incidence data from populationbased cancer registries in the US. We select the database 'Incidence - SEER 18 Regs Research Data' and extract the prostate cancer data for the county of San Bernardino in California during the period 2004-2014. We restrict to only white patients, aged 35-65 years old, with stage at diagnosis: localized, regional or distant and follow-up time greater than zero. Since a PSA level smaller than $4 \mathrm{ng} / \mathrm{ml}$ of blood is considered as normal and a PSA value between 4 and 10 $\mathrm{ng} / \mathrm{ml}$ is considered as a borderline range, we focus only on patients with PSA level greater than $10 \mathrm{ng} / \mathrm{ml}$. The event time is death because of prostate cancer. This cohort consists of 726 observations out of which 654 do not experience cancer related death (i.e. around $90 \%$ are censored). The follow-up time ranges from 2 to 155 months. For most of the patients the cancer has been diagnosed at early stage (localized), while for 228 of them the stage at diagnosis is 'regional' and only for 51 it is 'distant'. The PSA level varies from 10.1 to $94 \mathrm{ng} / \mathrm{ml}$, with median value $15.4 \mathrm{ng} / \mathrm{ml}$, mean value $21.9 \mathrm{ng} / \mathrm{ml}$ and standard deviation $16 \mathrm{ng} / \mathrm{ml}$. We use a logistic/Cox mixture cure model to analyse this dataset and the covariates of interest are the PSA level (continuous variable centered to the mean and measured with error) and stage at diagnosis. The latter one is classified using two dummy Bernoulli variables $S_{1}$ and $S_{2}$, indicating distant and regional stage respectively.

The use of the cure models is justified from the presence of a long plateau containing around $18 \%$ of the observations visible in the Kaplan-Meier curve ([17]) in Figure 1. Moreover, the Kaplan-Meier curves depending on stage at diagnosis in Figure 1 confirm that being in the distant stage significantly affects the probability of being cured. To see how sensitive the results are to the measurement error, we first estimate the model ignoring the measurement error ('naive') and then we apply the simex procedure with quadratic extrapolation function for two levels of measurement error, namely with standard deviation $v=4.8$ and $v=8$, corresponding to a ratio between the standard deviation of the error and the standard deviation of the covariate equal to 0.3 and 0.5 (we considered slightly smaller error than in the simulation setting in order to be closer to real life scenarios). In all three cases we use both the maximum likelihood estimation method and the presmoothing based method. The standard deviations of the estimates are computed through 1000 bootstrap samples. We consider such a large number of bootstrap samples because we noted that the estimated standard deviation for $\gamma_{3}$ (distant stage) is not very stable due to the 

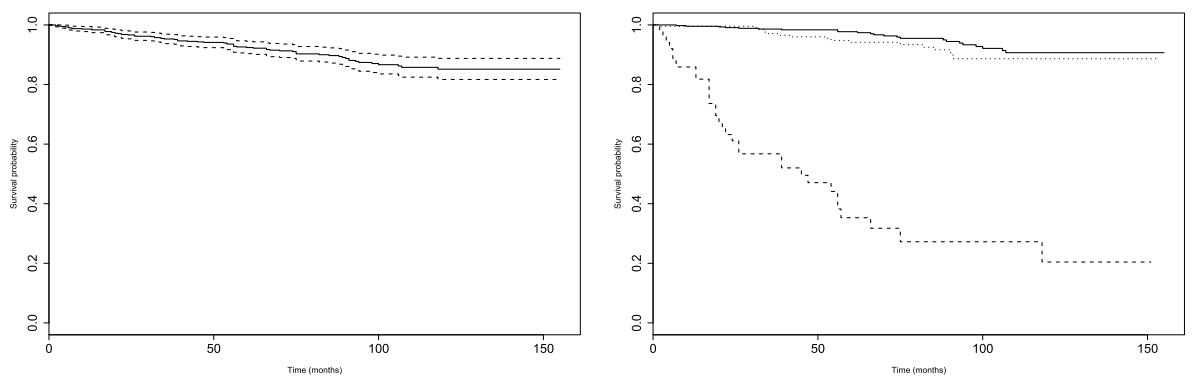

FIG 1. Left panel: Kaplan Meier survival curve for the prostate cancer data. Right panel: Kaplan Meier survival curves based on cancer stage at diagnosis, localized (solid), regional (dotted) and distant (dashed).

TABLE 5

Coefficient estimates, estimated standard deviations and p-values for the prostate cancer data using the naive and the simex method based on the maximum likelihood (1) and the presmoothing (2) approach.

\begin{tabular}{|c|c|c|c|c|c|c|c|c|c|}
\hline & & & \multicolumn{4}{|c|}{ incidence } & \multicolumn{3}{|c|}{ latency } \\
\hline & & & Intercept & PSA & $S_{1}$ & $S_{2}$ & PSA & $S_{1}$ & $S_{2}$ \\
\hline & & estimates & -2.2307 & 0.0302 & 3.2982 & 0.1021 & 0.0081 & 1.2775 & 0.6120 \\
\hline & $\stackrel{d}{d}$ & est. SD & 0.2043 & 0.0097 & 1.1943 & 0.3802 & 0.0078 & 0.5060 & 0.3485 \\
\hline & త్రే & p-value & 0.0000 & 0.0019 & 0.0058 & 0.7883 & 0.2999 & 0.0116 & 0.0790 \\
\hline & i & estimates & -2.3050 & 0.0293 & 3.2373 & 0.1909 & 0.0081 & 1.2600 & 0.5667 \\
\hline & $\Xi$ & est. SD & 0.2221 & 0.0084 & 0.4950 & 0.3780 & 0.0072 & 0.4937 & 0.3219 \\
\hline & 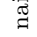 & p-value & 0.0000 & 0.0005 & 0.0000 & 0.6135 & 0.2619 & 0.0107 & 0.0852 \\
\hline \multirow{6}{*}{$\begin{array}{l}\infty \\
\underset{+}{+} \\
\| \\
2\end{array}$} & 方 & estimates & -2.2311 & 0.0306 & 3.2927 & 0.0990 & 0.0081 & 1.2757 & 0.6151 \\
\hline & d. & est. SD & 0.2048 & 0.0099 & 1.1939 & 0.3809 & 0.0079 & 0.5065 & 0.3492 \\
\hline & . ह्च & p-value & 0.0000 & 0.0019 & 0.0058 & 0.7949 & 0.3066 & 0.0118 & 0.0782 \\
\hline & $\stackrel{v}{u}$ & estimates & -2.2757 & 0.0281 & 3.2500 & 0.1779 & 0.0085 & 1.2658 & 0.5766 \\
\hline & $\ddot{x}$ & est. SD & 0.2937 & 0.0106 & 0.6480 & 0.5603 & 0.0073 & 0.4971 & 0.3405 \\
\hline & हี & p-value & 0.0000 & 0.0083 & 0.0000 & 0.6992 & 0.2442 & 0.0109 & 0.0903 \\
\hline \multirow{6}{*}{$\begin{array}{l}\infty \\
\| \\
2\end{array}$} & $\overrightarrow{1}$ & estimates & -2.2337 & 0.0317 & 3.2857 & 0.0995 & 0.0081 & 1.2836 & 0.6150 \\
\hline & de & est. SD & 0.2046 & 0.0099 & 1.1957 & 0.3814 & 0.0080 & 0.5064 & 0.3494 \\
\hline & .ี & p-value & 0.0000 & 0.0014 & 0.0060 & 0.7942 & 0.3092 & 00112 & 0.0784 \\
\hline & $\stackrel{v}{y}$ & estimates & -2.2752 & 0.0285 & 3.2412 & 0.2110 & 0.0086 & 1.2746 & 0.5655 \\
\hline & 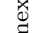 & est. SD & 0.2992 & 0.0110 & 0.6687 & 0.4712 & 0.0074 & 0.4996 & 0.3434 \\
\hline & . & p-value & 0.0000 & 0.0096 & 0.0000 & 0.6543 & 0.2444 & 0.0107 & 0.0997 \\
\hline
\end{tabular}

TABLE 6

Estimated cure probability for given PSA level and stage. The naive and simex estimators are computed using the maximum likelihood (1) or the presmoothing (2) approach.

\begin{tabular}{lrrrrrr}
\hline & \multicolumn{3}{c}{ 'Localized' } & \multicolumn{3}{c}{ 'Distant' } \\
PSA (ng/ml) & 10 & 22 & 34 & 10 & 22 & 34 \\
\hline naive - 1 & $93.0 \%$ & $90.3 \%$ & $86.7 \%$ & $33.1 \%$ & $25.6 \%$ & $19.3 \%$ \\
simex - 1 $(v=4.8)$ & $93.1 \%$ & $90.3 \%$ & $86.6 \%$ & $33.3 \%$ & $25.7 \%$ & $19.3 \%$ \\
simex - 1 $(v=8)$ & $93.2 \%$ & $90.3 \%$ & $86.4 \%$ & $33.8 \%$ & $25.9 \%$ & $19.3 \%$ \\
naive - 2 & $93.4 \%$ & $90.9 \%$ & $87.6 \%$ & $35.9 \%$ & $28.2 \%$ & $21.7 \%$ \\
simex - 2 $(v=4.8)$ & $93.2 \%$ & $90.7 \%$ & $87.4 \%$ & $34.6 \%$ & $27.4 \%$ & $21.2 \%$ \\
simex - 2 $(v=8)$ & $93.2 \%$ & $90.7 \%$ & $87.3 \%$ & $34.9 \%$ & $27.6 \%$ & $21.3 \%$ \\
\hline
\end{tabular}

small sample size of that category. The results are reported in Table 5.

First of all we observe that, independently of the estimation method that we 
use, the PSA level and being in the distant stage are significant for the cure probability, while only the latter one is significant for survival of uncured patients (at level 5\%). The positive sign of the coefficients confirms that high PSA level and distant stage are related to low cure probability and poor survival. Note that the estimated coefficient for the PSA value seems very small but it corresponds to a coefficient around 0.5 for the standardized variable. Given that the sample size is also large, we expect that, if the measurement error is relatively large, the use of the simex procedure would give more accurate results. Moreover, since there is some correlation between the PSA level and the stage of cancer, the measurement error might induce bias also in the other coefficients. For the maximum likelihood estimator, the estimated effect of the PSA level on the cure probability is slightly stronger when taking into account the measurement error, while the effect of the distant stage is slightly weakened. The opposite happens with the estimation based on presmoothing. To understand what these differences in the estimates mean in practical terms we compute the cure probability for patients with distant or localized stage and three different PSA levels: $10 \mathrm{ng} / \mathrm{ml}, 22 \mathrm{ng} / \mathrm{ml}$ (mean value) and $34 \mathrm{ng} / \mathrm{ml}$ (see Table 6).

Contrary to our expectation, we see that, in this example, there is not much difference between the naive and the simex approach. We observed in the simulation study that, when the bias induced by the measurement error is large, it is significantly reduced by the simex procedure and otherwise simex has little effect (see for example estimation of $\gamma_{2}$ in Model 1 and Model 2, Scenario 1 with $v=0.2$ or estimation of $\beta_{2}$ in Model 5 , Scenario 2 with $v_{2}=0.2$ ). Hence, we can conclude that in this example, the bias induced by the mismeasured PSA value is small. This is probably due to the fact that the effect of the PSA value on survival is weak (the absolute value of its coefficient is small compared to the intercept and the coefficient of $S_{1}$ ). The very high cure and censoring rate might also play a role. On the other hand, correlation between PSA and the stage of cancer would lead to induced bias even for the coefficients corresponding to $S_{1}$ and $S_{2}$. From the simulation study (see Model 5) we expect this bias to be of the same order as for the mismeasured covariate. Thus, since here the bias for the coefficient of the PSA value is small, even for the coefficients of $S_{1}$ and $S_{2}$ we do not observe much difference between the naive and simex method. Finally, we find that the estimated cure probabilities are larger when using the estimators based on presmoothing. Based again on the simulation study (cases with small bias), it is more likely that presmoothing behaves better than the maximum likelihood approach.

\section{Discussion}

In this paper we proposed a simulation-extrapolation procedure to correct for the bias induced by the measurement error in a mixture cure model. We focused on the logistic/Cox model and two existing estimation methods that can be incorporated in the simex algorithm. However, the proposed procedure and the asymptotic theory hold more in general for mixture cure models. Compared to 
promotion time models, mixture cure models allow us to separate the effect of the covariates on the cure probability and on survival. Simulations show that if the mismeasured covariate affects only one of these two components, the estimation of the other component remains undisturbed even if the variables are correlated. However, the use of the simex algorithm to correct for the bias, not always leads to better results in terms of mean squared error. The decision on whether to choose simex over the naive approach (ignoring the bias) depends on a number of factors. In particular, a large sample size, a strong effect of the covariate, a relatively large measurement error and low censoring favour the use of the simex approach. In practice, the effect of the covariates is not known in advance but, based on medical evidence for example, one has some prior knowledge about whether a covariate has a strong prognostic value (or at least not very weak compared to the other variables). In general, we would suggest that both methods (naive and simex) should be applied to check the sensitivity of the estimators. Results can then be compared with the simulation settings presented in this paper for getting a better understanding of the scenario in consideration.

A natural extension of this work would be to study the effect of the measurement error and simex method on the cumulative hazard, which would be of interest in estimating personalized survival curves. Here we only show consistency and asymptotic normality but did not investigate this further since the use of the simex procedure over many points of the support would be computationally intensive. In the context of nonparametric estimation, one could try to relax the parametric model of the incidence and consider for example a singleindex model instead. Furthermore, it would also be of interest to combine the simex procedure with variable selection procedures for situations in which the number of predictor variables is very large.

\section{Appendix A: Proofs}

Proof of Theorem 1. For a fixed $\lambda$ and $b$, from condition (A1) we have

$$
\left\|\hat{\gamma}_{\lambda, b}-\gamma_{\lambda}\right\| \rightarrow 0, \quad\left\|\hat{\beta}_{\lambda, b}-\beta_{\lambda}\right\| \rightarrow 0 \quad \text { and } \quad \sup _{t \in[0, \tau]}\left|\hat{\Lambda}_{\lambda, b}(t)-\Lambda_{\lambda}(t)\right| \rightarrow 0
$$

with probability one. By definition of $\hat{\gamma}_{\lambda}, \hat{\beta}_{\lambda}$ and $\hat{\Lambda}_{\lambda}$ as averages over the correspondent values for $b=1, \ldots, B((7))$ and Slutsky's theorem, it follows that for each $\lambda \in\left\{\lambda_{1}, \ldots, \lambda_{K}\right\}$

$$
\left\|\hat{\gamma}_{\lambda}-\gamma_{\lambda}\right\| \rightarrow 0, \quad\left\|\hat{\beta}_{\lambda}-\beta_{\lambda}\right\| \rightarrow 0 \quad \text { and } \quad \sup _{t \in[0, \tau]}\left|\hat{\Lambda}_{\lambda}(t)-\Lambda_{\lambda}(t)\right| \rightarrow 0
$$

almost surely. Next we first focus on consistency of $\hat{\gamma}_{\text {simex }}$. Since we are assuming that $g_{\gamma}\left(a_{\gamma}^{*}, \lambda\right)=\left(g_{\gamma, 1}\left(a_{\gamma_{1}}^{*}, \lambda\right), \ldots, g_{\gamma, p}\left(a_{\gamma_{p}}^{*}, \lambda\right)\right)^{T}$ is the true extrapolation function, we have $\gamma_{\lambda}=g_{\gamma}\left(a_{\gamma}^{*}, \lambda\right)$ and $\gamma^{*}=g_{\gamma}\left(a_{\gamma}^{*},-1\right)$. On the other hand, $\hat{\gamma}_{\text {simex }}=g_{\gamma}\left(\hat{a}_{\gamma},-1\right)$, where $\hat{a}_{\gamma}$ is the least squares estimator of $a_{\gamma}^{*}$, i.e. it solves

$$
\Psi_{n}\left(a_{\gamma}\right)=\dot{g}_{\gamma}\left(a_{\gamma}, \boldsymbol{\lambda}\right)^{T}\left\{g_{\gamma}\left(a_{\gamma}, \boldsymbol{\lambda}\right)-\hat{\gamma}_{\boldsymbol{\lambda}}\right\}=0
$$


where $\hat{\gamma}_{\boldsymbol{\lambda}}=\left(\hat{\gamma}_{\lambda_{1}}^{T}, \ldots, \hat{\gamma}_{\lambda_{K}}^{T}\right)^{T}, g_{\gamma}\left(a_{\gamma}, \boldsymbol{\lambda}\right)=\left(g_{\gamma}\left(a_{\gamma}, \lambda_{1}\right)^{T}, \ldots, g_{\gamma}\left(a_{\gamma}, \lambda_{K}\right)^{T}\right)^{T}$ and $\dot{g}_{\gamma}\left(a_{\gamma}, \boldsymbol{\lambda}\right)$ is the $p K \times p \operatorname{dim}\left(a_{\gamma}\right)$ matrix of partial derivatives of the elements of $g_{\gamma}\left(a_{\gamma}, \boldsymbol{\lambda}\right)$ with respect to the elements of $a_{\gamma}$. Moreover, the true parameters $a_{\gamma}^{*}$ are the solution of

$$
\Psi\left(a_{\gamma}\right)=\dot{g}_{\gamma}\left(a_{\gamma}, \boldsymbol{\lambda}\right)^{T}\left\{g_{\gamma}\left(a_{\gamma}, \boldsymbol{\lambda}\right)-\gamma_{\boldsymbol{\lambda}}\right\}=0
$$

and

$$
\sup _{a_{\gamma}}\left\|\Psi_{n}(a)-\Psi(a)\right\| \leq \sup _{a_{\gamma}}\left\|\dot{g}_{\gamma}\left(a_{\gamma}, \boldsymbol{\lambda}\right)\right\|\left\|\hat{\gamma}_{\boldsymbol{\lambda}}-\gamma_{\boldsymbol{\lambda}}\right\| \rightarrow 0 \quad \text { a.s. }
$$

Hence, if $a_{\gamma}^{*}$ is the unique solution of $\Psi\left(a_{\gamma}\right)=0$, it follows that $\hat{a}_{\gamma} \rightarrow a_{\gamma}^{*}$ with probability one. From the continuous mapping theorem it follows that

$$
\left\|\hat{\gamma}_{\text {simex }}-\gamma^{*}\right\| \rightarrow 0 \quad \text { a.s. }
$$

Consistency of $\hat{\beta}_{\text {simex }}$ can be proven in the same way. For the function $\hat{\Lambda}_{\text {simex }}$ we suppose that for every $t \in[0, \tau], \Lambda_{\lambda}(t)$ can be specified by a function $g_{\Lambda, t}\left(a_{t}, \lambda\right)$ depending on a parametric vector $a_{t}$ and $\Lambda^{*}(t)=g_{\Lambda, t}\left(a_{t},-1\right)$. Hence, arguing as above, for any fixed $t \in\left[0, \tau_{0}\right]$ we can show that

$$
\left|\hat{\Lambda}_{\text {simex }}(t)-\Lambda^{*}(t)\right| \rightarrow 0 \quad \text { a.s. }
$$

Uniform consistency on the compact $[0, \tau]$ follows from the fact that $\Lambda^{*}$ is continuous and $\Lambda_{\text {SIMEX }}$ is non-decreasing.

Proof of Theorem 2. For fixed $\lambda$ and $b$, from condition (C2) we have

$$
\begin{aligned}
& h_{2}^{T}\left(\hat{\gamma}_{\lambda, b}-\gamma_{\lambda}\right)+h_{3}^{T}\left(\hat{\beta}_{\lambda, b}-\beta_{\lambda}\right)+\int_{0}^{\tau} h_{1}(s) \mathrm{d}\left(\hat{\Lambda}_{\lambda, b}-\Lambda_{\lambda}\right)(s) \\
& =\frac{1}{n} \sum_{i=1}^{n} \Psi\left(Y_{i}, \Delta_{i}, W_{i, \lambda, b}, h_{1}, h_{2}, h_{3}\right)+o_{P}\left(n^{-1 / 2}\right)
\end{aligned}
$$

uniformly over $\left(h_{1}, h_{2}, h_{3}\right) \in \mathcal{H}_{\mathfrak{m}}$. As a result,

$$
\begin{aligned}
& h_{2}^{T}\left(\hat{\gamma}_{\lambda}-\gamma_{\lambda}\right)+h_{3}^{T}\left(\hat{\beta}_{\lambda}-\beta_{\lambda}\right)+\int_{0}^{\tau} h_{1}(s) \mathrm{d}\left(\hat{\Lambda}_{\lambda}-\Lambda_{\lambda}\right)(s) \\
& =h_{2}^{T}\left(\frac{1}{B} \sum_{b=1}^{B} \hat{\gamma}_{\lambda, b}-\gamma_{\lambda}\right)+h_{3}^{T}\left(\frac{1}{B} \sum_{b=1}^{B} \hat{\beta}_{\lambda, b}-\beta_{\lambda}\right) \\
& \quad+\int_{0}^{\tau} h_{1}(s) \mathrm{d}\left(\frac{1}{B} \sum_{b=1}^{B} \hat{\Lambda}_{\lambda, b}-\Lambda_{\lambda}\right)(s) \\
& =\frac{1}{n} \sum_{i=1}^{n}\left\{\frac{1}{B} \sum_{b=1}^{B} \Psi\left(Y_{i}, \Delta_{i}, W_{i, \lambda, b}, h_{1}, h_{2}, h_{3}\right)\right\}+o_{P}\left(n^{-1 / 2}\right) .
\end{aligned}
$$

Since sum of Donsker classes is Donsker (see Lemma 2.10.6 in [36]), it follows that the process

$$
n^{1 / 2}\left\{h_{2}^{T}\left(\hat{\gamma}_{\lambda}-\gamma_{\lambda}\right)+h_{3}^{T}\left(\hat{\beta}_{\lambda}-\beta_{\lambda}\right)+\int_{0}^{\tau} h_{1}(s) \mathrm{d}\left(\hat{\Lambda}_{\lambda}-\Lambda_{\lambda}\right)(s)\right\}
$$


converges weakly to a zero-mean Gaussian process $G_{\lambda}$ indexed by $h=\left(h_{1}, h_{2}\right.$, $\left.h_{3}\right) \in \mathcal{H}_{\mathfrak{m}}$ and covariance function

$$
\begin{aligned}
& \operatorname{Cov}\left(G_{\lambda}\left(h_{1}, h_{2}, h_{3}\right), G_{\lambda}\left(h_{1}^{*}, h_{2}^{*}, h_{3}^{*}\right)\right) \\
& =\mathbb{E}\left[\left\{\frac{1}{B} \sum_{b=1}^{B} \Psi\left(Y, \Delta, W_{\lambda, b}, h_{1}, h_{2}, h_{3}\right)\right\}\left\{\frac{1}{B} \sum_{b=1}^{B} \Psi\left(Y, \Delta, W_{\lambda, b}, h_{1}^{*}, h_{2}^{*}, h_{3}^{*}\right)\right\}\right] .
\end{aligned}
$$

Moreover, the $K$ dimensional vector

$$
n^{1 / 2}\left\{\begin{array}{c}
h_{2}^{T}\left(\hat{\gamma}_{\lambda_{1}}-\gamma_{\lambda_{1}}\right)+h_{3}^{T}\left(\hat{\beta}_{\lambda_{1}}-\beta_{\lambda_{1}}\right)+\int_{0}^{\tau} h_{1}(s) \mathrm{d}\left(\hat{\Lambda}_{\lambda_{1}}-\Lambda_{\lambda_{1}}\right)(s) \\
\vdots \\
h_{2}^{T}\left(\hat{\gamma}_{\lambda_{K}}-\gamma_{\lambda_{K}}\right)+h_{3}^{T}\left(\hat{\beta}_{\lambda_{K}}-\beta_{\lambda_{K}}\right)+\int_{0}^{\tau} h_{1}(s) \mathrm{d}\left(\hat{\Lambda}_{\lambda_{K}}-\Lambda_{\lambda_{K}}\right)(s)
\end{array}\right\}
$$

converges to a $K$ dimensional Gaussian process $G_{\boldsymbol{\lambda}}$ with mean zero and covariance function between the $i$ th and the $j t h$ component

$$
\mathbb{E}\left[\left\{\frac{1}{B} \sum_{b=1}^{B} \Psi\left(Y, \Delta, W_{\lambda_{i}, b}, h_{1}, h_{2}, h_{3}\right)\right\}\left\{\frac{1}{B} \sum_{b=1}^{B} \Psi\left(Y, \Delta, W_{\lambda_{j}, b}, h_{1}^{*}, h_{2}^{*}, h_{3}^{*}\right)\right\}\right] .
$$

In particular, if we take $h_{1} \equiv 0, h_{3}=0$ and $h_{2}=(0, \ldots, 0,1,0, \ldots, 0)$ with $h_{2}$ containing 1 at the $j$ th position $(j=1, \ldots, p)$ and 0 elsewhere, we obtain the weak convergence of $n^{1 / 2}\left(\hat{\gamma}_{\boldsymbol{\lambda}}-\gamma_{\boldsymbol{\lambda}}\right)$ to a multivariate normal random variable with mean zero and covariance matrix $\Sigma_{\gamma, \boldsymbol{\lambda}}$. With the same reasoning we also obtain $n^{1 / 2}\left(\hat{\beta}_{\boldsymbol{\lambda}}-\beta_{\boldsymbol{\lambda}}\right) \stackrel{d}{\rightarrow} N\left(0, \Sigma_{\beta, \boldsymbol{\lambda}}\right)$. For $\hat{\Lambda}_{\boldsymbol{\lambda}}$ we consider the class

$$
\left\{\left(h_{1}, h_{2}, h_{3}\right) \in \mathcal{H}_{\mathfrak{m}}: h_{2}=h_{3}=0 \text { and } h_{1}(s)=\mathbb{1}_{\{s \leq t\}}, t \in[0, \tau]\right\}
$$

and obtain the weak convergence of $n^{1 / 2}\left\{\hat{\Lambda}_{\boldsymbol{\lambda}}(t)-\Lambda_{\boldsymbol{\lambda}}(t)\right\}$ to a Gaussian process $\mathcal{G}_{\boldsymbol{\lambda}}$ indexed by $t \in[0, \tau]$.

Next we prove the asymptotic normality of $\hat{\gamma}_{\text {simex }}$. Since we are assuming that $g_{\gamma}\left(a_{\gamma}^{*}, \lambda\right)=\left(g_{\gamma, 1}\left(a_{\gamma_{1}}^{*}, \lambda\right), \ldots, g_{\gamma, p}\left(a_{\gamma_{p}}^{*}, \lambda\right)\right)^{T}$ is the true extrapolation function, we have $\gamma_{\lambda}=g_{\gamma}\left(a_{\gamma}^{*}, \lambda\right)$ and $\gamma^{*}=g_{\gamma}\left(a_{\gamma}^{*},-1\right)$. On the other hand, $\hat{\gamma}_{\text {simex }}=$ $g_{\gamma}\left(\hat{a}_{\gamma},-1\right)$, where $\hat{a}_{\gamma}$ is the least squares estimator of $a_{\gamma}^{*}$, i.e. it solves

$$
\Psi_{n}\left(a_{\gamma}\right)=\dot{g}_{\gamma}\left(a_{\gamma}, \boldsymbol{\lambda}\right)^{T}\left\{g_{\gamma}\left(a_{\gamma}, \boldsymbol{\lambda}\right)-\hat{\gamma}_{\boldsymbol{\lambda}}\right\}=0
$$

where $\hat{\gamma}_{\boldsymbol{\lambda}}=\left(\hat{\gamma}_{\lambda_{1}}^{T}, \ldots, \hat{\gamma}_{\lambda_{K}}^{T}\right)^{T}, g_{\gamma}\left(a_{\gamma}, \boldsymbol{\lambda}\right)=\left(g_{\gamma}\left(a_{\gamma}, \lambda_{1}\right)^{T}, \ldots, g_{\gamma}\left(a_{\gamma}, \lambda_{K}\right)^{T}\right)^{T}$ and $\dot{g}_{\gamma}\left(a_{\gamma}, \boldsymbol{\lambda}\right)$ is the $p K \times p \operatorname{dim}\left(a_{\gamma}\right)$ matrix of partial derivatives of the elements of $g_{\gamma}\left(a_{\gamma}, \boldsymbol{\lambda}\right)$ with respect to the elements of $a_{\gamma}$. Since $\hat{a}_{\gamma}$ solves equation (10) and $\hat{a}_{\gamma} \rightarrow a_{\gamma}^{*}$ with probability one (see proof of Theorem 1), if $\dot{g}_{\gamma}\left(a_{\gamma}, \boldsymbol{\lambda}\right)$ is bounded and continuous w.r.t. $a_{\gamma}$ and $\dot{g}_{\gamma}\left(a_{\gamma}, \boldsymbol{\lambda}\right)^{T} \dot{g}_{\gamma}\left(a_{\gamma}, \boldsymbol{\lambda}\right)$ is invertible, we have

$$
n^{1 / 2}\left(\hat{a}_{\gamma}-a_{\gamma}^{*}\right)=\left\{\dot{g}_{\gamma}\left(a_{\gamma}^{*}, \boldsymbol{\lambda}\right)^{T} \dot{g}_{\gamma}\left(a_{\gamma}^{*}, \boldsymbol{\lambda}\right)\right\}^{-1} \dot{g}_{\gamma}\left(a_{\gamma}^{*}, \boldsymbol{\lambda}\right)^{T} n^{1 / 2}\left(\hat{\gamma}_{\boldsymbol{\lambda}}-\gamma_{\boldsymbol{\lambda}}\right)+o_{P}(1) .
$$

As a result,

$$
n^{1 / 2}\left(\hat{a}_{\gamma}-a_{\gamma}^{*}\right) \stackrel{d}{\rightarrow}\left\{\dot{g}_{\gamma}\left(a_{\gamma}^{*}, \boldsymbol{\lambda}\right)^{T} \dot{g}_{\gamma}\left(a_{\gamma}^{*}, \boldsymbol{\lambda}\right)\right\}^{-1} \dot{g}_{\gamma}\left(a_{\gamma}^{*}, \boldsymbol{\lambda}\right)^{T} N\left(0, \Sigma_{\gamma, \boldsymbol{\lambda}}\right) .
$$


Finally, using the delta method, we obtain

$$
n^{1 / 2}\left(\hat{\gamma}_{\text {simex }}-\gamma^{*}\right) \stackrel{d}{\rightarrow} \dot{g}_{\gamma}\left(a_{\gamma}^{*},-1\right)\left\{\dot{g}_{\gamma}\left(a_{\gamma}^{*}, \boldsymbol{\lambda}\right)^{T} \dot{g}_{\gamma}\left(a_{\gamma}^{*}, \boldsymbol{\lambda}\right)\right\}^{-1} \dot{g}_{\gamma}\left(a_{\gamma}^{*}, \boldsymbol{\lambda}\right)^{T} N\left(0, \Sigma_{\gamma, \boldsymbol{\lambda}}\right),
$$

meaning that $n^{1 / 2}\left(\hat{\gamma}_{\text {simex }}-\gamma^{*}\right)$ converges weakly to a multivariate normal random variable with mean zero and covariance matrix

$$
\begin{aligned}
\Sigma_{\gamma}=\dot{g}_{\gamma}\left(a_{\gamma}^{*},-1\right) & \left\{\dot{g}_{\gamma}\left(a_{\gamma}^{*}, \boldsymbol{\lambda}\right)^{T} \dot{g}_{\gamma}\left(a_{\gamma}^{*}, \boldsymbol{\lambda}\right)\right\}^{-1} \dot{g}_{\gamma}\left(a_{\gamma}^{*}, \boldsymbol{\lambda}\right)^{T} \\
& \times \Sigma_{\gamma, \boldsymbol{\lambda}} \dot{g}_{\gamma}\left(a_{\gamma}^{*}, \boldsymbol{\lambda}\right)\left\{\dot{g}_{\gamma}\left(a_{\gamma}^{*}, \boldsymbol{\lambda}\right)^{T} \dot{g}_{\gamma}\left(a_{\gamma}^{*}, \boldsymbol{\lambda}\right)\right\}^{-1} \dot{g}_{\gamma}\left(a_{\gamma}^{*},-1\right)^{T} .
\end{aligned}
$$

In the same way it can be shown that $n^{1 / 2}\left(\hat{\beta}_{\text {simex }}-\beta^{*}\right)$ converges weakly to a multivariate normal random variable with mean zero and covariance matrix

$$
\begin{aligned}
\Sigma_{\beta}=\dot{g}_{\beta}\left(a_{\beta}^{*},-1\right)\{ & \left\{\dot{g}_{\beta}\left(a_{\beta}^{*}, \boldsymbol{\lambda}\right)^{T} \dot{g}_{\beta}\left(a_{\beta}^{*}, \boldsymbol{\lambda}\right)\right\}^{-1} \dot{g}_{\beta}\left(a_{\beta}^{*}, \boldsymbol{\lambda}\right)^{T} \\
& \times \Sigma_{\beta, \boldsymbol{\lambda}} \dot{g}_{\beta}\left(a_{\beta}^{*}, \boldsymbol{\lambda}\right)\left\{\dot{g}_{\beta}\left(a_{\beta}^{*}, \boldsymbol{\lambda}\right)^{T} \dot{g}_{\beta}\left(a_{\beta}^{*}, \boldsymbol{\lambda}\right)\right\}^{-1} \dot{g}_{\beta}\left(a_{\beta}^{*},-1\right)^{T} .
\end{aligned}
$$

Similarly, for the nonparametric component we have

$n^{1 / 2}\left(\hat{a}_{t}-a_{t}^{*}\right)=\left\{\dot{g}_{\Lambda, t}\left(a_{t}^{*}, \boldsymbol{\lambda}\right)^{T} \dot{g}_{\Lambda, t}\left(a_{t}^{*}, \boldsymbol{\lambda}\right)\right\}^{-1} \dot{g}_{\Lambda, t}\left(a_{t}^{*}, \boldsymbol{\lambda}\right)^{T} n^{1 / 2}\left(\hat{\Lambda}_{\boldsymbol{\lambda}}(t)-\Lambda_{\boldsymbol{\lambda}}(t)\right)+o_{P}(1)$

for all $t \in[0, \tau]$. From the weak convergence of the process $n^{1 / 2}\left(\hat{\Lambda}_{\boldsymbol{\lambda}}-\Lambda_{\boldsymbol{\lambda}}\right)$, it follows that $n^{1 / 2}\left(\hat{a}_{t}-a_{t}^{*}\right)$ converges in distribution to the Gaussian process

$$
\left\{\dot{g}_{\Lambda, t}\left(a_{t}^{*}, \boldsymbol{\lambda}\right)^{T} \dot{g}_{\Lambda, t}\left(a_{t}^{*}, \boldsymbol{\lambda}\right)\right\}^{-1} \dot{g}_{\Lambda, t}\left(a_{t}^{*}, \boldsymbol{\lambda}\right)^{T} \mathcal{G}_{\boldsymbol{\lambda}} .
$$

Once more, the delta method yields that $n^{1 / 2}\left(\hat{\Lambda}_{\text {simex }}-\Lambda_{0}\right)$ converges weakly to the Gaussian process

$$
\mathcal{G}=\dot{g}_{\Lambda, t}\left(a_{t}^{*},-1\right)\left\{\dot{g}_{\Lambda, t}\left(a_{t}^{*}, \boldsymbol{\lambda}\right)^{T} \dot{g}_{\Lambda, t}\left(a_{t}^{*}, \boldsymbol{\lambda}\right)\right\}^{-1} \dot{g}_{\Lambda, t}\left(a_{t}^{*}, \boldsymbol{\lambda}\right)^{T} \mathcal{G}_{\boldsymbol{\lambda}} .
$$

In order to establish the asymptotic results of the maximum likelihood estimator in the logistic/Cox mixture cure model, the following conditions are required in [22]:

1) The function $\Lambda^{*}(t)$ is strictly increasing and continuously differentiable, and $\Lambda^{*}(\tau)<\infty$, where $\tau$ denotes the total follow-up of the study.

2) $\theta^{*}=\left(\gamma^{*}, \beta^{*}\right)$ lies in the interior of a compact set and the covariate vectors $\mathrm{Z}$ and $\mathrm{X}$ are bounded in the sense that $\mathbb{P}(\|Z\|<m$ and $\|X\|<m)=1$ for some constant $m>0$.

3) There exists a positive constant $\epsilon$ such that $\mathbb{P}\left(C \geq T_{0} \geq \tau \mid Z, X\right)>\epsilon$ with probability one.

4) $\mathbb{P}(Y \geq t \mid Z, X)$ is continuous in $\mathrm{t}$.

Because of the model identifiability conditions (I2') and (I3') in Section 4.1, in this case we must have $\tau=\tau_{0}$, i.e. the support of $T_{0}$ coincides with the total follow-up, and $\mathbb{P}\left(T_{0}=\tau \mid Z, X\right)>\epsilon$.

For the estimator based on presmoothing, in [26] the asymptotic results are obtained only for the case with one continuous covariate for the incidence component. The following assumptions are required: 
(AC2) The parameters $\beta^{*}$ and $\gamma^{*}$ lie in the interior of compact sets $B \subset \mathbb{R}^{q}$, $G \subset \mathbb{R}^{2}$.

(AC5) The covariates are bounded: $\mathbb{P}(\|Z\|<m$ and $|X|<m)=1$ for some $m>0$.

(AC6) The function $\lambda^{*}(t)$ is strictly positive and continuous on $\left[0, \tau_{0}\right)$.

(AC7) With probability one, the conditional distribution function of the censoring times $F_{C}(t \mid x, z)$ is continuous in $t$ on $\left[0, \tau_{0}\right]$ and there exists a constant $K>0$ such that

$$
\inf _{0 \leq t_{1}<t_{2} \leq \tau_{0}} \inf _{x, z}\left\{F_{C}\left(t_{2} \mid x, z\right)-F_{C}\left(t_{1} \mid x, z\right)\right\}>K\left(t_{2}-t_{1}\right) .
$$

(C1) The bandwidth $b$ is such that $n b^{4} \rightarrow 0$ and $n b^{3+\xi} /\left(\log b^{-1}\right) \rightarrow \infty$ for some $\xi>0$.

(C2) The support $\mathcal{X}$ of $X$ is a compact subset of $\mathbb{R}$. The density $f_{X}(\cdot)$ of $X$ is bounded away from zero and twice differentiable with bounded second derivative.

(C3) The kernel $k$ is a twice continuously differentiable, symmetric probability density function with compact support and $\int u k(u) \mathrm{d} u=0$.

(C4) (i) The functions $H([0, t] \mid x), H_{1}([0, t] \mid x)$, where

$$
H_{k}([0, t] \mid x)=\mathbb{P}(Y \leq t, \Delta=k \mid X=x), \quad k \in\{0,1\}
$$

and $H([0, t] \mid x)=H_{1}([0, t] \mid x)+H_{0}([0, t] \mid x)$, are twice differentiable with respect to $x$, with uniformly bounded derivatives for all $t \leq \tau_{0}, x \in \mathcal{X}$. Moreover, there exist continuous nondecreasing functions $L_{1}, L_{2}, L_{3}$ such that $L_{i}(0)=0, L_{i}\left(\tau_{0}\right)<\infty$ and for all $t, s \in\left[0, \tau_{0}\right], x \in \mathcal{X}$,

$$
\begin{aligned}
\left|H_{c}(t \mid x)-H_{c}(s \mid x)\right| & \leq\left|L_{1}(t)-L_{1}(s)\right|, \\
\left|H_{1 c}(t \mid x)-H_{1 c}(s \mid x)\right| & \leq\left|L_{1}(t)-L_{1}(s)\right| \\
\left|\frac{\partial H_{c}(t \mid x)}{\partial x}-\frac{\partial H_{c}(s \mid x)}{\partial x}\right| & \leq\left|L_{2}(t)-L_{2}(s)\right| \\
\left|\frac{\partial H_{1 c}(t \mid x)}{\partial x}-\frac{\partial H_{1 c}(s \mid x)}{\partial x}\right| & \leq\left|L_{3}(t)-L_{3}(s)\right|,
\end{aligned}
$$

where the subscript $\mathrm{c}$ denotes the continuous part of a function.

(ii) The jump points for the distribution function $F_{C}(t \mid x)$ of the censoring times given the covariate, are finite and the same for all $x$. The partial derivative of $F_{C}(t \mid x)$ with respect to $x$ exists and is uniformly bounded for all $t \leq \tau_{0}, x \in \mathcal{X}$. Moreover, the partial derivative with respect to $x$ of $F(t \mid x)$ (distribution function of the survival times $T$ given $X=x$ ) exists and is uniformly bounded for all $t \leq \tau_{0}, x \in \mathcal{X}$.

(C5) The survival time $T$ and the censoring time $C$ are independent given $X$.

The results can be generalized to cases with additional discrete covariates in $X$ with a finite number of atoms.

In what follows, we assume such conditions to be satisfied in order to use the results of [22] and [26] for the naive estimator. We show that these naive 
estimators satisfy our conditions (A1)-(A2) and as a result, the simex estimators have the desired asymptotic properties.

Proof of Theorem 3. Let $\Upsilon_{0}=\left(\gamma^{*}, \beta^{*}, \Lambda^{*}\right), \theta_{0}=\left(\gamma^{*}, \beta^{*}\right), \hat{\theta}_{n}=\left(\hat{\gamma}_{n}, \hat{\beta}_{n}\right)$ and $\mathcal{H}_{\mathfrak{m}}$ as in (A2). Define the continuous linear operator $\sigma=\left(\sigma_{1}, \sigma_{2}\right)$ from $\mathcal{H}_{\mathfrak{m}}$ to $\mathcal{H}_{\mathfrak{m}}$ of the form

$$
\begin{aligned}
\sigma_{1}(h)(t)= & \mathbb{E}\left[\mathbb{1}_{\{Y \geq t\}} V\left(t, \Upsilon_{0}\right)(h) g\left(t, \Upsilon_{0}\right) e^{\beta_{0}^{T} Z}\right] \\
& -\mathbb{E}\left[\int_{t}^{\tau} \mathbb{1}_{\{Y \geq s\}} V\left(t, \Upsilon_{0}\right)(h) g\left(s, \Upsilon_{0}\right)\left\{1-g\left(s, \Upsilon_{0}\right)\right\} e^{2 \beta^{* T} Z} \mathrm{~d} \Lambda^{*}(s)\right]
\end{aligned}
$$

and

$$
\sigma_{2}(h)(t)=\mathbb{E}\left[\int_{0}^{\tau} \mathbb{1}_{\{Y \geq t\}} W\left(t, \Upsilon_{0}\right) V\left(t, \Upsilon_{0}\right)(h) g\left(t, \Upsilon_{0}\right) e^{\beta^{* T} Z} \mathrm{~d} \Lambda^{*}(t)\right]
$$

where

$$
g(t, \Lambda, \beta, \gamma)=\frac{\phi(\gamma, X) \exp \left(-\Lambda(t) \exp \left(\beta^{T} Z\right)\right)}{1-\phi(\gamma, X)+\phi(\gamma, X) \exp \left(-\Lambda(t) \exp \left(\beta^{T} Z\right)\right)},
$$

$V\left(t, \Upsilon_{0}\right)(h)=h_{1}(t)-\left\{1-g\left(t, \Upsilon_{0}\right)\right\} e^{\beta^{* T} Z} \int_{0}^{t} h_{1}(s) \mathrm{d} \Lambda^{*}(s)+\left(h_{2}^{T}, h_{3}^{T}\right) W\left(t, \Upsilon_{0}\right)$ and

$$
W\left(t, \Upsilon_{0}\right)=\left(\left\{1-g\left(t, \Upsilon_{0}\right)\right\} X^{T},\left[1-\left\{1-g\left(t, \Upsilon_{0}\right)\right\} e^{\beta^{* T} Z} \Lambda^{*}(t)\right] Z^{T}\right)^{T} .
$$

Note that in our case $X=\left(W_{\lambda}^{(1)}, W_{\lambda}^{(2)}\right)$ and $Z=\left(W_{\lambda}^{(1)}, W_{\lambda}^{(2)}\right)$. In the proof of Theorem 2 in [22] (page 572) it is shown that

$$
\begin{aligned}
& \int_{0}^{\tau} \sigma_{1}(h)(t) \mathrm{d} \sqrt{n}\left(\Lambda_{n}-\Lambda^{*}\right)(t)+\sqrt{n}\left(\hat{\theta}_{n}-\theta_{0}\right)^{T} \sigma_{2}(h) \\
& =\sqrt{n}\left\{S_{n}\left(\Upsilon_{0}\right)-S\left(\Upsilon_{0}\right)\right\}(h)+o_{P}(1),
\end{aligned}
$$

where

$$
\sqrt{n}\left\{S_{n}\left(\Upsilon_{0}\right)-S\left(\Upsilon_{0}\right)\right\}\left(h_{1}, h_{2}, h_{3}\right)=\int f_{h}(y, \delta, x, z) \mathrm{d} \sqrt{n}\left(\mathbb{P}_{n}-\mathbb{P}\right)(y, \delta, x, z)
$$

and $\left\{f_{h}(y, \delta, x, z), h \in \mathcal{H}_{\mathfrak{m}}\right\}$ is a uniformly bounded Donsker class such that

$$
\mathrm{E}\left[f_{h}(Y, \Delta, X, Z)\right]=S\left(\Upsilon_{0}\right)=0 .
$$

In [22] it is also shown that $\sigma$ is invertible with inverse $\sigma^{-1}=\left(\sigma_{1}^{-1}, \sigma_{2}^{-1}\right)$. Hence, for all $h \in \mathcal{H}_{\mathfrak{m}}$, if in (17) we replace $h$ by $\sigma^{-1}(h)$, we obtain

$$
\begin{aligned}
& \int_{0}^{\tau} h_{1}(t) \mathrm{d}\left(\Lambda_{n}(t)-\Lambda^{*}(t)\right)+h_{2}^{T}\left(\hat{\gamma}_{n}-\gamma^{*}\right)+h_{3}^{T}\left(\hat{\beta}_{n}-\beta^{*}\right) \\
& =\int f_{\sigma^{-1}(h)}(y, \delta, x, z) \mathrm{d}\left(\mathbb{P}_{n}-\mathbb{P}\right)(y, \delta, x, z)+o_{P}\left(n^{-1 / 2}\right)
\end{aligned}
$$


and (A2) holds with

$$
\Psi_{\lambda}\left(y, \delta, w, h_{1}, h_{2}, h_{3}\right)=f_{\sigma^{-1}(h)}\left(y, \delta,\left(w^{(1)}, w^{(2)}\right),\left(w^{(2)}, w^{(3)}\right)\right)
$$

Proof of Theorem 4. In [26] it is shown that

$$
\hat{\gamma}_{n}-\gamma^{*}=-\left(\Gamma_{1}^{T} \Gamma_{1}\right)^{-1} \Gamma_{1}^{T} \int \psi(y, \delta, x)\left(\mathbb{P}_{n}-\mathbb{P}\right)(y, \delta, x, z)+o_{P}\left(n^{-1 / 2}\right)
$$

(see their equation (A.33)), where

$$
\begin{gathered}
\Gamma_{1}=-\mathrm{E}\left[\left\{\frac{1}{\phi\left(\gamma^{*}, X\right)}+\frac{1}{1-\phi\left(\gamma^{*}, X\right)}\right\} \nabla_{\gamma} \phi\left(\gamma^{*}, X\right) \nabla_{\gamma} \phi\left(\gamma^{*}, X\right)^{T}\right], \\
\psi(y, \delta, x)=-\left\{\frac{\Delta \mathbb{1}_{\left\{y \leq \tau_{0}\right\}}}{1-H(y \mid x)}-\int_{0}^{y \wedge \tau_{0}} \frac{H_{1}(d s \mid x)}{(1-H(s \mid x))^{2}}\right\} \frac{1}{\phi\left(\gamma^{*}, x\right)} \nabla_{\gamma} \phi\left(\gamma^{*}, x\right)
\end{gathered}
$$

with $H_{k}(t \mid x)=\mathbb{P}(Y \leq t, \Delta=k \mid X=x)$ for $k=0,1$ and $H(t \mid x)=H_{1}(t \mid x)+$ $H_{0}(t \mid x)$. Moreover we have $\mathrm{E}[\psi(Y, \Delta, X)]=0$.

Let $\Upsilon_{0}=\left(\gamma^{*}, \beta^{*}, \Lambda^{*}\right)$ and $\tilde{\mathcal{H}}_{\mathfrak{m}}=\left\{\tilde{h}=\left(h_{1}, h_{3}\right) \in B V\left[0, \tau_{0}\right] \times \mathbb{R}^{q}:\left\|h_{1}\right\|_{v}+\right.$ $\left.\left\|h_{3}\right\|_{L_{1}} \leq \mathfrak{m}\right\}$. Define the continuous linear operator $\sigma=\left(\sigma_{1}, \sigma_{2}\right)$ from $\tilde{\mathcal{H}}_{\mathfrak{m}}$ to $\tilde{\mathcal{H}}_{\mathfrak{m}}$ as in (14), (15) with

$$
V\left(t, \Upsilon_{0}\right)(h)=h_{1}(t)-\left\{1-g\left(t, \Upsilon_{0}\right)\right\} e^{\beta^{* T} Z} \int_{0}^{t} h_{1}(s) \mathrm{d} \Lambda^{*}(s)+h_{3}^{T} W\left(t, \Upsilon_{0}\right)
$$

and

$$
W\left(t, \Upsilon_{0}\right)=\left[1-\left\{1-g\left(t, \Upsilon_{0}\right)\right\} e^{\beta^{* T} Z} \Lambda^{*}(t)\right] Z .
$$

From equations (A37)-(A38) in [26] we have

$$
\begin{aligned}
& \int_{0}^{\tau} \sigma_{1}(\tilde{h})(t) \mathrm{d} \sqrt{n}\left(\Lambda_{n}-\Lambda^{*}\right)(t)+\sqrt{n}\left(\hat{\beta}_{n}-\beta^{*}\right)^{T} \sigma_{2}(\tilde{h}) \\
& =\sqrt{n}\left\{\hat{S}_{n}\left(\Upsilon_{0}\right)-S\left(\Upsilon_{0}\right)\right\}(\tilde{h})+o_{P}(1),
\end{aligned}
$$

where

$$
\sqrt{n}\left\{\hat{S}_{n}\left(\Upsilon_{0}\right)-S\left(\Upsilon_{0}\right)\right\}\left(h_{1}, h_{3}\right)=\int \tilde{f}_{\tilde{h}}(y, \delta, x, z) \mathrm{d} \sqrt{n}\left(\mathbb{P}_{n}-\mathbb{P}\right)(y, \delta, x, z)
$$

for some uniformly bounded Donsker class $\left\{f_{\tilde{h}}(y, \delta, x, z), \tilde{h} \in \tilde{\mathcal{H}}_{\mathfrak{m}}\right\}$ with

$$
\mathrm{E}\left[\tilde{f}_{\tilde{h}}(Y, \Delta, X, Z)\right]=0 .
$$

Hence, if we replace $\tilde{h}$ by $\sigma^{-1}(\tilde{h})$, we obtain

$$
\begin{aligned}
& \int_{0}^{\tau} h_{1}(t) \mathrm{d}\left(\Lambda_{n}(t)-\Lambda^{*}(t)\right)+h_{3}^{T}\left(\hat{\beta}_{n}-\beta^{*}\right) \\
& =\int \tilde{f}_{\sigma^{-1}(\tilde{h})}(y, \delta, x, z) \mathrm{d}\left(\mathbb{P}_{n}-\mathbb{P}\right)(y, \delta, x, z)+o_{P}\left(n^{-1 / 2}\right) .
\end{aligned}
$$


Note that in our case $x=\left(w_{\lambda}^{(1)}, w_{\lambda}^{(2)}\right)$ and $z=\left(w_{\lambda}^{(1)}, w_{\lambda}^{(2)}\right)$. Moreover, if $h=$ $\left(h_{1}, h_{2}, h_{3}\right) \in \mathcal{H}_{\mathfrak{m}}$, then $\tilde{h}=\left(h_{1}, h_{3}\right) \in \tilde{\mathcal{H}}_{\mathfrak{m}}$. It follows that (A2) holds with

$$
\begin{aligned}
\Psi_{\lambda}\left(y, \delta, w, h_{1}, h_{2}, h_{3}\right)= & -h_{2}^{T}\left(\Gamma_{1}^{T} \Gamma_{1}\right)^{-1} \Gamma_{1}^{T} \psi\left(y, \delta,\left(w^{(1)}, w^{(2)}\right)\right) \\
& +\tilde{f}_{\sigma^{-1}\left(\left(h_{1}, h_{3}\right)\right)}\left(y, \delta,\left(w^{(1)}, w^{(2)}\right),\left(w^{(2)}, w^{(3)}\right)\right)
\end{aligned}
$$

\section{Appendix B: Additional simulation results}

TABLE 7

Bias, variance and MSE of $\hat{\gamma}$ and $\hat{\beta}$ for the naive and simex method using the maximum likelihood (1) and the presmoothing (2) approach for Model 1 ( $n=400)$. The first column

\begin{tabular}{|c|c|c|c|c|c|c|c|c|c|c|c|c|c|}
\hline \multirow{5}{*}{$\overline{1 / 1 / 1}$} & \multirow[b]{2}{*}{ Par. } & \multicolumn{3}{|c|}{ naive - 1} & \multicolumn{3}{|c|}{ naive -2} & \multicolumn{3}{|c|}{ simex - 1} & \multicolumn{3}{|c|}{ simex -2} \\
\hline & & Bias & Var. & MSE & Bias & Var. & MSE & Bias & Var. & MSE & Bias & Var. & MSE \\
\hline & $\gamma_{1}$ & 0.8 & 1.9 & 1.9 & 0.5 & 1.8 & 1.8 & 0.9 & 1.9 & 1.9 & 0.7 & 1.9 & 1.9 \\
\hline & $\gamma_{2}$ & 4.1 & 1.4 & 1.5 & -3.7 & 1.3 & 1.4 & -1.8 & 2.5 & 2.5 & -1.7 & 2.5 & 2.5 \\
\hline & $\beta$ & -44.3 & 0.3 & 19.9 & -44.3 & 0.3 & 20.0 & -20.1 & 0.9 & 4.9 & -20.2 & 0.9 & 4.9 \\
\hline \multirow[t]{3}{*}{$1 / 1 / 2$} & $\gamma_{1}$ & 1.8 & 2.7 & 2.7 & 0.5 & 2.6 & 2.6 & 1.5 & 2.6 & 2.6 & 0.7 & 3.0 & 3.0 \\
\hline & 1 & -4.1 & 2.0 & 2.1 & -1.8 & 2.0 & 2.0 & -1.3 & 3.6 & 3.6 & 1.0 & 4. & 4.3 \\
\hline & $\beta$ & -43.2 & 0.4 & 19.1 & -43.5 & 0.4 & 19.3 & -19.2 & 1.1 & 4.8 & -19.4 & 1. & 4.9 \\
\hline \multirow[t]{3}{*}{$1 / 2 / 1$} & $\gamma_{1}$ & -0.3 & 1.3 & 1.3 & -0.6 & 1.3 & 1.3 & -0.4 & 1.3 & 1.3 & -0.5 & 1.3 & 1.3 \\
\hline & $\gamma_{2}$ & -3.7 & 0.8 & 0.9 & -3.8 & 0.7 & 0.9 & -1.5 & 1.5 & 1.5 & -1.8 & 1.4 & 1.4 \\
\hline & $\beta$ & -43.9 & 0.6 & 19.9 & -43.9 & 0.4 & 19.3 & -19.8 & 1.6 & 5.5 & -19.8 & 1. & 5.5 \\
\hline \multirow[t]{3}{*}{$1 / 2 / 2$} & $\gamma_{1}$ & -0.2 & 2.1 & 2.1 & -1.4 & 2.1 & 2.1 & -0.5 & 2.1 & 2.1 & -1.4 & 2. & 2.3 \\
\hline & $\gamma_{2}$ & -3.1 & 1.3 & 1.4 & -2.3 & 1.2 & 1.3 & -0.5 & 2.4 & 2.4 & 0.3 & ? & .5 \\
\hline & $\beta$ & 42.5 & 0.9 & 19.0 & -42.8 & 0.9 & 19.3 & -18.6 & 2.4 & 5.9 & -18.9 & & 5.9 \\
\hline \multirow[t]{3}{*}{$2 / 1 / 1$} & $\gamma_{1}$ & -2.0 & 1.8 & 1.9 & -2.3 & 1.8 & 1.8 & -0.4 & 1.5 & .9 & -0.8 & 1 & 1.9 \\
\hline & $\gamma_{2}$ & 7.3 & 1.2 & 4.2 & -17.9 & 1.2 & 4.4 & -5.7 & 2. & & -6.8 & & 2.9 \\
\hline & $\beta$ & -45.0 & 0.3 & 20.6 & -45.0 & 0.3 & 20.6 & -20.8 & 0.9 & 2 & -20.8 & 0 & 5.2 \\
\hline \multirow{3}{*}{$2 / 1 / 2$} & $\gamma_{1}$ & 1.2 & 2.3 & 2.3 & -2.3 & 2.3 & 2.3 & 0.3 & 2. & 2.4 & -0.7 & 2. & 2.5 \\
\hline & $\gamma_{2}$ & 17.2 & 1. & 4.7 & -16.7 & 1.6 & 4.4 & -5.2 & 3. & 6 & -5.3 & 3 & 3.8 \\
\hline & $\beta$ & 44.2 & 0.4 & 19.9 & -44.3 & 0.4 & 20.0 & -20.1 & 1. & 1 & -20.1 & & .1 \\
\hline \multirow[t]{3}{*}{$2 / 2 / 1$} & $\gamma_{1}$ & -0.5 & 1.3 & 1.3 & -0.8 & 1.3 & 1.3 & -0.5 & 1. & 1.3 & -0.7 & 1. & 1.4 \\
\hline & 1 & -17.3 & 0 . & 3. & -18.4 & 0. & 4.2 & -5.6 & 1. & & -7.5 & & 2.3 \\
\hline & $\beta$ & 44.5 & 0.6 & 20.4 & -44.4 & 0.6 & 20.4 & -20.0 & 1. & 5.7 & -19.9 & 1. & 5.6 \\
\hline \multirow[t]{3}{*}{$2 / 2 / 2$} & $\gamma_{1}$ & 0.0 & 2.4 & 2.4 & -1.5 & 2.3 & 2.4 & -0.2 & 2. & 4 & -1.5 & 2 & 2.7 \\
\hline & $\gamma_{2}$ & 17.1 & 1. & 4.4 & -17.6 & 1.6 & 4.7 & -5.1 & 3.0 & 3.3 & -5.9 & 3. & 3.9 \\
\hline & $\beta$ & 42.8 & 1. & 19 & -42.9 & & & -18.4 & & & -18.6 & & 6.1 \\
\hline \multirow[t]{3}{*}{$3 / 1 / 1$} & $\gamma_{1}$ & -37.0 & 3. & 17.1 & -38.7 & 3.2 & 18.2 & -16.3 & 5.9 & 8.6 & -18.8 & 5. & 9.1 \\
\hline & $\gamma$ & 7.5 & 2. & 79.2 & -90.4 & & & -38.3 & 7.1 & & -43.1 & 6.4 & 24.9 \\
\hline & $\beta$ & 48.9 & 0. & 24.3 & -48.8 & 0.4 & 24.2 & -24.1 & 1.1 & 6.9 & -24.0 & 1.1 & 6.9 \\
\hline \multirow[t]{3}{*}{$3 / 1 / 2$} & $\gamma_{1}$ & -35.3 & 4.8 & 17.3 & -38.1 & 4. & 19.4 & -14.0 & 8.7 & 10.7 & -17.6 & 10.0 & 13.1 \\
\hline & $\gamma_{2}$ & 87.6 & 3. & 80.4 & -90.5 & 3.9 & 85.8 & -37.8 & 10.3 & 24.6 & -43.0 & 11.8 & 30.4 \\
\hline & $\beta$ & -4 & 0. & 23. & -47.4 & & 23.0 & -22.6 & & & -22.5 & & 6.3 \\
\hline \multirow[t]{3}{*}{$3 / 2 / 1$} & $\gamma_{1}$ & -1.1 & 1.7 & 1. & -1.5 & 1.6 & 1.6 & -1.0 & 2.2 & 2.2 & -1.7 & 2.1 & 2.1 \\
\hline & $\gamma$ & & 2. & 83. & -9 & 1 & 89.8 & -41.2 & 5.5 & 22.4 & -46.3 & 3 & 26.7 \\
\hline & $\beta$ & 51.5 & 0. & 27.2 & -51.3 & 0. & 27.1 & -26.6 & 2.1 & 9.2 & -26.5 & 2.1 & 9.1 \\
\hline \multirow[t]{3}{*}{$3 / 2 / 2$} & $\gamma_{1}$ & 0.0 & 2. & 2. & -1.7 & & 2.5 & 0.1 & & 3. & -1.5 & 0 & 3.6 \\
\hline & 11 & 91.0 & 3.2 & 85.9 & -95.4 & 3.0 & 94.1 & -41.9 & 8.9 & 26.5 & -49.1 & 8.7 & 32.9 \\
\hline & $\beta$ & -49.9 & 1.2 & 26.1 & -49.5 & 1.2 & 25.7 & -25.1 & 3.1 & 9.4 & -24.6 & 3.0 & 9.0 \\
\hline
\end{tabular}
gives the setting/scenario/cens. level. All numbers were multiplied by 100. 
TABLE 8

Bias, variance and MSE of $\hat{\gamma}$ and $\hat{\beta}$ for the naive and simex method based on the maximum likelihood (1) or the presmoothing (2) approach in Models 2-5 $(n=200)$. The first column gives the model, scenario and the standard deviation of the measurement error. All numbers were multiplied by 100 .

\begin{tabular}{|c|c|c|c|c|c|c|c|c|c|c|c|c|c|}
\hline \multirow[b]{2}{*}{ Mod./Scen./v } & \multirow[b]{2}{*}{ Par. } & \multicolumn{3}{|c|}{ naive - 1} & \multicolumn{3}{|c|}{ naive -2} & \multicolumn{3}{|c|}{ simex - 1} & \multicolumn{3}{|c|}{ simex - 2} \\
\hline & & Bias & Var. & MSE & Bias & Var. & MSE & Bias & Var. & MSE & Bias & Var. & MSE \\
\hline \multirow[t]{5}{*}{$2 / 3 / 0.2$} & $\gamma_{1}$ & 0.0 & 7.3 & 7.3 & 0.0 & 7.3 & 7.3 & -0.9 & 7.7 & 7.7 & -0.6 & 8.0 & 8.0 \\
\hline & $\gamma_{2}$ & -10.3 & 12.7 & 13.8 & -16.7 & 12.0 & 14.8 & 5.4 & 16.7 & 17.0 & -1.3 & 18.0 & 18.0 \\
\hline & $\gamma_{3}$ & 0.5 & 14.4 & 14.4 & -0.7 & 14.3 & 14.3 & 2.5 & 15.2 & 15.3 & 1.1 & 16.1 & 16.1 \\
\hline & $\beta_{1}$ & -8.9 & 6.3 & 7.0 & -8.4 & 6.2 & 6.9 & 1.5 & 9.0 & 9.0 & 2.0 & 8.9 & 9.0 \\
\hline & $\beta_{2}$ & -0.6 & 7.2 & 7.2 & -0.6 & 7.2 & 7.2 & 0.7 & 7.6 & 7.6 & 0.7 & 7.5 & 7.6 \\
\hline \multirow[t]{5}{*}{$2 / 3 / 0.4$} & $\gamma_{1}$ & 1.7 & 6.8 & 6.8 & 1.9 & 7.0 & 7.1 & -0.1 & 7.6 & 7.6 & 0.9 & 9.0 & 9.0 \\
\hline & $\gamma_{2}$ & -45.2 & 9.2 & 29.6 & -51.5 & 8.4 & 35.0 & -8.0 & 19.5 & 20.1 & -16.5 & 19.8 & 22.5 \\
\hline & $\gamma_{3}$ & -3.2 & 13.3 & 13.4 & -4.6 & 13.5 & 13.7 & 0.9 & 15.1 & 15.1 & -1.3 & 17.0 & 17.1 \\
\hline & $\beta_{1}$ & -29.2 & 4.2 & 12.8 & -28.8 & 4.2 & 12.5 & -9.5 & 9.3 & 10.2 & -8.9 & 9.3 & 10.0 \\
\hline & $\beta_{2}$ & -3.1 & 7.2 & 7.3 & -3.1 & 7.2 & 7.3 & -0.6 & 8.0 & 8.0 & -0.5 & 8.0 & 8.0 \\
\hline \multirow[t]{5}{*}{$3 / 1 / 0.1$} & $\gamma_{1}$ & 5.4 & 11.2 & 11.5 & 4.2 & 11.3 & 11.5 & 5.4 & 11.2 & 11.5 & 4.2 & 11.3 & 11.5 \\
\hline & $\gamma_{2}$ & 3.0 & 20.4 & 20.5 & -0.1 & 20.8 & 20.8 & 3.0 & 10.4 & 20.5 & -0.1 & 20.8 & 20.8 \\
\hline & $\gamma_{3}$ & 0.5 & 24.9 & 24.9 & -1.0 & 26.3 & 26.3 & 0.5 & 24.9 & 24.9 & -1.0 & 26.3 & 26.3 \\
\hline & $\beta_{1}$ & 0.9 & 4.3 & 4.3 & 1.0 & 4.3 & 4.3 & 1.1 & 4.3 & 4.3 & 1.1 & 4.3 & 4.3 \\
\hline & $\beta_{2}$ & -5.8 & 9.6 & 9.9 & -6.0 & 9.5 & 9.9 & -0.9 & 12.1 & 12.1 & 1.1 & 12.1 & 12.1 \\
\hline \multirow[t]{5}{*}{$3 / 1 / 0.2$} & $\gamma_{1}$ & 5.4 & 11.2 & 11.4 & 4.2 & 11.3 & 11.5 & 5.4 & 11.2 & 11.4 & 4.2 & 11.3 & 11.5 \\
\hline & $\gamma_{2}$ & 2.8 & 20.4 & 20.4 & -0.2 & 20.9 & 20.9 & 2.9 & 20.4 & 20.5 & -0.2 & 20.9 & 20.9 \\
\hline & $\gamma_{3}$ & 0.4 & 24.8 & 24.8 & -1.0 & 26.2 & 26.2 & 0.3 & 24.8 & 24.8 & -1.0 & 26.2 & 26.2 \\
\hline & $\beta_{1}$ & 0.6 & 4.3 & 4.3 & 0.6 & 4.2 & 4.2 & 0.9 & 4.4 & 4.4 & 0.9 & 4.3 & 4.3 \\
\hline & $\beta_{2}$ & -16.6 & 7.2 & 9.9 & -16.7 & 7.2 & 10.0 & -5.6 & 13.4 & 13.7 & -5.8 & 13.3 & 13.6 \\
\hline \multirow[t]{5}{*}{$3 / 2 / 0.1$} & $\gamma_{1}$ & 4.4 & 7.3 & 7.5 & 3.6 & 7.2 & 7.4 & 4.3 & 7.3 & 7.5 & 3.6 & 7.2 & 7.4 \\
\hline & $\gamma_{2}$ & 3.3 & 12.0 & 12.1 & -0.5 & 11.8 & 11.8 & 3.3 & 12.0 & 12.1 & -0.5 & 11.8 & 11.8 \\
\hline & $\gamma_{3}$ & -2.5 & 12.5 & 12.5 & -2.4 & 12.3 & 12.3 & -2.5 & 12.5 & 12.5 & -2.4 & 12.3 & 12.3 \\
\hline & $\beta_{1}$ & 0.5 & 3.7 & 3.7 & 0.6 & 3.7 & 3.7 & 1.1 & 3.7 & 3.7 & 1.2 & 3.7 & 3.7 \\
\hline & $\beta_{2}$ & 8.3 & 9.0 & 9.7 & 8.3 & 9.0 & 9.7 & -1.8 & 11.6 & 11.6 & -1.8 & 11.6 & 11.6 \\
\hline \multirow[t]{5}{*}{$3 / 2 / 0.2$} & $\gamma_{1}$ & 4.3 & 7.3 & 7.5 & 3.6 & 7.2 & 7.4 & 4.3 & 7.3 & 7.5 & 3.6 & 7.2 & 7.4 \\
\hline & $\gamma_{2}$ & 3.3 & 12.0 & 12.1 & -0.5 & 11.8 & 11.8 & 3.3 & 12.0 & 12.1 & -0.5 & 11.8 & 11.8 \\
\hline & $\gamma_{3}$ & -2.5 & 12.4 & 12.5 & -2.4 & 12.3 & 12.3 & -2.5 & 12.5 & 12.1 & -2.4 & 12.3 & 12.3 \\
\hline & $\beta_{1}$ & -0.8 & 3.7 & 3.7 & -0.7 & 3.7 & 3.7 & 0.5 & 3.9 & 3.9 & 0.6 & 3.9 & 3.9 \\
\hline & $\beta_{2}$ & 30.4 & 6.8 & 16.1 & 30.4 & 6.8 & 16.1 & 7.3 & 13.1 & 13.6 & 7.3 & 13.0 & 13.6 \\
\hline & $\gamma_{1}$ & 3.4 & 5.3 & 5.4 & 1.6 & 5.1 & 5.2 & 4.3 & 5.5 & 5.7 & 2.4 & 5.6 & 5.6 \\
\hline$v_{1}=0.35$ & $\gamma_{2}$ & -2.2 & 4.9 & 4.9 & -5.1 & 4.5 & 4.8 & 3.8 & 6.4 & 6.5 & 0.2 & 6.7 & 6.7 \\
\hline \multirow[t]{2}{*}{$v_{2}=0.2$} & $\beta_{1}$ & -6.5 & 1.0 & 1.5 & -6.3 & 1.0 & 1.4 & -0.5 & 1.4 & 1.4 & -0.3 & 1.4 & 1.4 \\
\hline & $\beta_{2}$ & 0.1 & 2.6 & 2.6 & 0.1 & 2.6 & 2.6 & 1.5 & 3.4 & 3.5 & 1.5 & 3.4 & 3.4 \\
\hline & $\gamma_{1}$ & 1.8 & 5.2 & 5.3 & 0.1 & 5.2 & 5.2 & 3.6 & 5.7 & 5.8 & 2.0 & 6.2 & 6.2 \\
\hline$v_{1}=0.7$ & $\gamma_{2}$ & -14.3 & 3.6 & 5.6 & -16.7 & 3.4 & 6.2 & -1.4 & 7.0 & 7.0 & -4.8 & 7.4 & 7.7 \\
\hline \multirow[t]{2}{*}{$v_{2}=0.4$} & $\beta_{1}$ & -18.8 & 0.8 & 4.3 & -18.7 & 0.8 & 4.3 & -6.8 & 1.7 & 2.1 & -6.7 & 1.7 & 2.1 \\
\hline & $\beta_{2}$ & -2.7 & 2.0 & 2.1 & -2.7 & 2.0 & 2.1 & 0.0 & 3.9 & 3.9 & 0.0 & 3.9 & 3.9 \\
\hline $4 / 2$ & $\gamma_{1}$ & -5.1 & 6.4 & 6.6 & -9.7 & 5.9 & 6.9 & 4.3 & 8.7 & 8.9 & -1.7 & 8.2 & 8.2 \\
\hline$v_{1}=0.3$ & $\gamma_{2}$ & -27.5 & 8.8 & 16.4 & -39.6 & 8.0 & 23.7 & 4.5 & 15.9 & 16.1 & -11.6 & 15.1 & 16.5 \\
\hline$v_{2}=0.2$ & $\beta_{1}$ & -1.4 & 1.2 & 1.2 & -1.3 & 1.2 & 1.2 & 0.1 & 1.7 & 1.7 & 0.3 & 1.7 & 1.7 \\
\hline & $\beta_{2}$ & -3.6 & 2.6 & 2.8 & -3.6 & 2.6 & 2.8 & 2.0 & 3.5 & 3.6 & 2.0 & 3.5 & 3.6 \\
\hline $4 / 2$ & $\gamma_{1}$ & -21.6 & 4.9 & 9.6 & -24.7 & 4.6 & 10.7 & -7.2 & 8.1 & 8.6 & -11.9 & 7.5 & 8.9 \\
\hline & $\gamma_{2}$ & -85.1 & 4.5 & 76.9 & -93.0 & 4.2 & 90.7 & -33.7 & 12.6 & 23.9 & -46.0 & 11.9 & 33.1 \\
\hline & $\beta_{1}$ & -4.4 & 0.8 & 1.0 & -4.2 & 0.8 & 1.0 & -1.8 & 1.8 & 1.8 & -1.6 & 1.7 & 1.8 \\
\hline & $\beta_{2}$ & 15.5 & 1.9 & 4.3 & -15.5 & 1.9 & 4.3 & -3.2 & 3.9 & 4.0 & -3.2 & 3.9 & 4.0 \\
\hline $5 / 3 / 0.39$ & $\gamma_{1}$ & 1.2 & 7.0 & 7.0 & -0.8 & 6.6 & 6.6 & 1.0 & 6.9 & 6.9 & -0.8 & 6.6 & 6.6 \\
\hline & & 11.4 & 20.3 & 21.6 & 3.8 & 20.9 & 21.1 & 11.7 & 20.5 & 21.8 & 3.8 & 20.9 & 21.1 \\
\hline & $\beta_{1}$ & 34.0 & 8.8 & 20.3 & 34.5 & 8.8 & 20.7 & 14.1 & 11.9 & 13.9 & 14.8 & 11.9 & 14.1 \\
\hline & $\beta_{2}$ & 38.4 & 4.7 & 19.4 & 38.8 & 4.7 & 19.7 & 13.1 & 10.7 & 12.4 & 13.6 & 10.7 & 12.5 \\
\hline $5 / 3 / 0.78$ & $\gamma_{1}$ & 1.5 & 7.2 & 7.2 & -0.8 & 6.6 & 6.6 & 1.3 & 7.2 & 7.2 & -0.8 & 6.6 & 6.6 \\
\hline & $\gamma_{2}$ & 11.0 & 20.5 & 21.7 & 3.8 & 20.9 & 21.1 & 11.2 & 20.7 & 22.0 & 3.8 & 20.9 & 21.1 \\
\hline & $\beta_{1}$ & 60.3 & 7.7 & 44.0 & 60.6 & 7.7 & 44.4 & 45.5 & 10.1 & 30.8 & 46.0 & 10.1 & 31.2 \\
\hline & $\beta_{2}$ & 72.0 & 2.1 & 54.0 & 72.2 & 2.1 & 54.2 & 53.4 & 6.6 & 35.2 & 53.7 & 6.6 & 35.5 \\
\hline
\end{tabular}


TABLE 9

Bias, variance and MSE of $\hat{\gamma}$ and $\hat{\beta}$ for the simex method based on the maximum likelihood

(1) or the presmoothing (2) approach with three different extrapolation functions. All numbers were multiplied by 100.

\begin{tabular}{rrrrrrrrrrrrrrr}
\hline & \multicolumn{1}{c}{$v=0.2$} & \multicolumn{1}{c}{ simex - 1} & \multicolumn{1}{c}{$v=0$} \\
& \multicolumn{1}{c}{ sar. } & Bias & Var. & MSE & Bias & Var. & MSE & Bias & Var. & MSE & Bias & Var. & MSE \\
\hline linear & $\gamma_{1}$ & 5.6 & 12.3 & 12.6 & 3.6 & 12.0 & 12.1 & 3.3 & 12.2 & 12.3 & 1.3 & 12.3 & 12.3 \\
& $\gamma_{2}$ & 1.9 & 21.9 & 22.0 & -3.5 & 19.8 & 20.0 & -18.2 & 18.7 & 22.0 & -22.9 & 17.0 & 22.3 \\
& $\gamma_{3}$ & -0.9 & 25.4 & 25.4 & -1.6 & 26.0 & 26.1 & -1.0 & 25.7 & 25.7 & -2.2 & 25.5 & 25.5 \\
& $\beta_{1}$ & -1.7 & 3.6 & 3.7 & -1.5 & 3.6 & 3.6 & -17.9 & 2.9 & 6.2 & -17.8 & 2.9 & 6.0 \\
& $\beta_{2}$ & 0.3 & 4.3 & 4.3 & 0.3 & 4.2 & 4.2 & -0.7 & 4.3 & 4.4 & -0.6 & 4.3 & 4.3 \\
cubic & $\gamma_{1}$ & 6.5 & 12.7 & 13.1 & 5.3 & 16.7 & 16.9 & 5.6 & 13.5 & 13.8 & 4.1 & 22.6 & 22.7 \\
& $\gamma_{2}$ & 5.9 & 28.1 & 28.4 & -0.5 & 34.2 & 34.2 & 0.5 & 35.4 & 35.4 & -4.4 & 54.4 & 54.6 \\
& $\gamma_{3}$ & -1.1 & 25.3 & 25.3 & -2.2 & 39.2 & 39.2 & -0.8 & 26.6 & 26.6 & -2.8 & 46.0 & 46.1 \\
& $\beta_{1}$ & 2.3 & 5.0 & 5.0 & 2.6 & 4.9 & 5.0 & -2.4 & 6.3 & 6.4 & -2.2 & 6.3 & 6.4 \\
& $\beta_{2}$ & 0.2 & 4.5 & 4.5 & 0.3 & 4.4 & 4.4 & -0.1 & 5.0 & 5.0 & 0.0 & 5.0 & 5.0 \\
\hline
\end{tabular}

TABLE 10

Bias, variance and MSE of $\hat{\gamma}$ and $\hat{\beta}$ for the simex method based on the maximum likelihood

(1) or the presmoothing (2) approach when the error distribution is misspecified. All numbers were multiplied by 100.

\begin{tabular}{|c|c|c|c|c|c|c|c|c|c|c|c|c|c|}
\hline & \multirow[b]{3}{*}{ Par. } & \multicolumn{6}{|c|}{$v=0.2$} & \multicolumn{6}{|c|}{$v=0.4$} \\
\hline & & \multicolumn{3}{|c|}{ simex -1} & \multicolumn{3}{|c|}{ simex -2} & \multicolumn{3}{|c|}{ simex -1} & \multicolumn{3}{|c|}{ simex -2} \\
\hline & & Bias & Var. & MSE & Bias & Var. & MSE & Bias & Var. & MSE & Bias & Var. & MSE \\
\hline \multirow[t]{5}{*}{ t-distr. } & $\gamma_{1}$ & 6.3 & 12.4 & 12.7 & 3.9 & 13.6 & 13.7 & 5.3 & 12.7 & 12.9 & 2.5 & 14.3 & 14.4 \\
\hline & $\gamma_{2}$ & 5.9 & 23.2 & 23.6 & 0.5 & 25.8 & 25.9 & -1.3 & 27.0 & 27.1 & -5.2 & 26.5 & 26.7 \\
\hline & $\gamma_{3}$ & -1.5 & 25.3 & 25.4 & -0.1 & 31.2 & 31.2 & -2.1 & 26.0 & 26.0 & -1.6 & 33.8 & 33.8 \\
\hline & $\beta_{1}$ & 0.1 & 4.1 & 4.1 & 0.3 & 4.0 & 4.0 & -8.7 & 4.8 & 5.6 & -8.5 & 4.8 & 5.5 \\
\hline & $\beta_{2}$ & 0.5 & 4.3 & 4.3 & 0.3 & 4.3 & 4.3 & 0.2 & 4.6 & 4.6 & 0.1 & 4.6 & 4.6 \\
\hline \multirow[t]{5}{*}{ Unif. } & $\gamma_{1}$ & 6.2 & 12.4 & 12.7 & 4.9 & 14.0 & 14.2 & 5.1 & 12.6 & 12.9 & 4.5 & 15.0 & 15.2 \\
\hline & $\gamma_{2}$ & 4.9 & 22.4 & 22.6 & -1.5 & 24.5 & 24.5 & -5.8 & 23.3 & 23.6 & -11.2 & 27.1 & 28.3 \\
\hline & $\gamma_{3}$ & -1.2 & 25.1 & 25.1 & -0.2 & 29.4 & 29.4 & -1.5 & 25.4 & 25.4 & -0.8 & 29.0 & 29.0 \\
\hline & $\beta_{1}$ & 0.1 & 4.0 & 4.0 & 0.4 & 4.0 & 4.0 & -9.5 & 4.3 & 5.2 & -9.2 & 4.2 & 5.0 \\
\hline & $\beta_{2}$ & 0.3 & 4.3 & 4.3 & 0.2 & 4.4 & 4.4 & -0.2 & 4.7 & 4.7 & -0.3 & 4.6 & 4.6 \\
\hline \multirow[t]{5}{*}{$\chi^{2}$} & $\gamma_{1}$ & 6.9 & 12.7 & 13.2 & 3.2 & 13.1 & 13.2 & 8.2 & 14.6 & 15.3 & 4.1 & 14.8 & 14.9 \\
\hline & $\gamma_{2}$ & 6.8 & 25.6 & 26.1 & -2.4 & 25.6 & 25.7 & 5.6 & 38.6 & 38.9 & -13.5 & 33.8 & 35.7 \\
\hline & $\gamma_{3}$ & -1.5 & 25.1 & 25.1 & -0.5 & 27.7 & 27.7 & -2.0 & 25.9 & 25.9 & -1.4 & 33.1 & 33.1 \\
\hline & $\beta_{1}$ & -0.5 & 4.5 & 4.5 & -0.1 & 4.4 & 4.4 & -12.9 & 6.0 & 7.7 & -11.8 & 5.9 & 7.3 \\
\hline & $\beta_{2}$ & 0.2 & 4.3 & 4.3 & 0.1 & 4.4 & 4.4 & -0.5 & 4.8 & 4.8 & -0.5 & 4.8 & 4.8 \\
\hline
\end{tabular}

TABLE 11

Bias, variance and MSE of $\hat{\gamma}$ and $\hat{\beta}$ for the simex method based on the maximum likelihood (1) or the presmoothing (2) approach when the error variance is misspecified. All numbers were multiplied by 100 .

\begin{tabular}{|c|c|c|c|c|c|c|c|c|c|c|c|c|c|}
\hline \multirow[b]{3}{*}{$v_{E}$} & \multirow[b]{3}{*}{ Par. } & \multicolumn{6}{|c|}{$v=0.2$} & \multicolumn{6}{|c|}{$v=0.4$} \\
\hline & & \multicolumn{3}{|c|}{ simex -1} & \multicolumn{3}{|c|}{ simex -2} & \multicolumn{3}{|c|}{ simex - 1} & \multicolumn{3}{|c|}{ simex -2} \\
\hline & & Bias & Var. & MSE & Bias & Var. & MSE & Bias & Var. & MSE & Bias & Var. & MSE \\
\hline \multirow[t]{5}{*}{$\overline{v-0.1}$} & $\gamma_{1}$ & 5.0 & 12.2 & 12.4 & 3.3 & 12.1 & 12.2 & 3.5 & 12.6 & 12.7 & 1.6 & 13.7 & 13.8 \\
\hline & $\gamma_{2}$ & -3.7 & 19.9 & 20.0 & -8.9 & 18.4 & 19.2 & -16.4 & 20.9 & 23.6 & -21.2 & 22.6 & 27.1 \\
\hline & $\gamma_{3}$ & -1.0 & 25.1 & 25.1 & -2.2 & 27.3 & 27.4 & -0.9 & 25.7 & 25.7 & -2.1 & 28.6 & 28.6 \\
\hline & $\beta_{1}$ & -6.1 & 3.2 & 3.6 & -5.9 & 3.1 & 3.5 & -16.1 & 3.3 & 5.9 & -15.9 & 3.3 & 5.8 \\
\hline & $\beta_{2}$ & 0.0 & 4.2 & 4.2 & 0.0 & 4.2 & 4.2 & -0.6 & 4.4 & 4.4 & -0.5 & 4.4 & 4.4 \\
\hline \multirow{5}{*}{$v+0.1$} & $\gamma_{1}$ & 7.4 & 13.4 & 13.9 & 6.0 & 14.0 & 14.4 & 5.5 & 13.8 & 14.1 & 3.9 & 15.6 & 15.8 \\
\hline & $\gamma_{2}$ & 18.6 & 32.7 & 36.1 & 12.2 & 32.1 & 33.6 & 5.5 & 34.2 & 34.5 & 0.1 & 35.7 & 35.7 \\
\hline & $\gamma_{3}$ & -0.4 & 26.1 & 26.1 & -1.5 & 31.3 & 31.3 & -0.3 & 27.1 & 27.1 & -1.7 & 31.4 & 31.4 \\
\hline & $\beta_{1}$ & 12.4 & 5.5 & 7.1 & 12.6 & 5.5 & 7.1 & 1.1 & 5.6 & 5.6 & 1.3 & 5.5 & 5.5 \\
\hline & $\beta_{2}$ & 1.2 & 4.6 & 4.6 & 1.3 & 4.6 & 4.6 & 0.6 & 4.9 & 4.9 & 0.7 & 4.9 & 4.9 \\
\hline
\end{tabular}




\section{References}

[1] Amico, M. and Van Keilegom, I. (2018). Cure models in survival analysis. Annual Review of Statistics and Its Application 5, 311-342.

[2] Amico, M., Van Keilegom, I., and Legrand, C. (2019). The singleindex/Cox mixture cure model. Biometrics 75, 2, 452-462.

[3] Berkson, J. and Gage, R. P. (1952). Survival curve for cancer patients following treatment. Journal of the American Statistical Association 47, 259, 501-515.

[4] Bertrand, A., Legrand, C., Carroll, R. J., De Meester, C., AND VAN KeIlegom, I. (2017). Inference in a survival cure model with mismeasured covariates using a simulation-extrapolation approach. Biometrika 104, 1, 31-50.

[5] Bertrand, A., Legrand, C., Léonard, D., and Van Keilegom, I. (2017). Robustness of estimation methods in a survival cure model with mismeasured covariates. Computational Statistics \& Data Analysis 113, $3-18$.

[6] Bertrand, A., Van Keilegom, I., and Legrand, C. (2019). Flexible parametric approach to classical measurement error variance estimation without auxiliary data. Biometrics 75, 1, 297-307.

[7] BoAG, J. W. (1949). Maximum likelihood estimates of the proportion of patients cured by cancer therapy. Journal of the Royal Statistical Society. Series B (Methodological) 11, 1, 15-53.

[8] Burke, K. And Patilea, V. (2020). A likelihood-based approach for cure regression models. TEST, 1-20.

[9] Cai, C., Zou, Y., Peng, Y., and Zhang, J. (2012). smcure: An Rpackage for estimating semiparametric mixture cure models. Computer methods and programs in biomedicine 108, 3, 1255-1260.

[10] Carroll, R. J., Küchenhoff, H., Lombard, F., and Stefanski, L. A. (1996). Asymptotics for the simex estimator in nonlinear measurement error models. Journal of the American Statistical Association 91, 433, 242250.

[11] Carroll, R. J., Ruppert, D., Stefanski, L. A., and Crainiceanu, C. M. (2006). Measurement error in nonlinear models: a modern perspective. CRC press.

[12] Chen, L.-P. (2019). Semiparametric estimation for cure survival model with left-truncated and right-censored data and covariate measurement error. Statistics \& Probability Letters.

[13] Cook, J. R. And Stefanski, L. A. (1994). Simulation-extrapolation estimation in parametric measurement error models. Journal of the American Statistical association 89, 428, 1314-1328.

[14] Cox, D. R. (1972). Regression models and life-tables. Journal of the Royal Statistical Society. Series B. Methodological 34, 187-220.

[15] Dirick, L., Claeskens, G., and Baesens, B. (2015). An akaike information criterion for multiple event mixture cure models. European Journal of Operational Research 241, 2, 449-457. 
[16] Greene, W. F. and Cai, J. (2004). Measurement error in covariates in the marginal hazards model for multivariate failure time data. Biometrics 60, 4, 987-996.

[17] Kaplan, E. L. And Meier, P. (1958). Nonparametric estimation from incomplete observations. Journal of the American statistical association 53, 282, 457-481.

[18] Legrand, C. And Bertrand, A. (2019). Cure models in oncology clinical trials. Textb Clin Trials Oncol Stat Perspect 1, 465-492.

[19] Li, C.-S. AND TAYLOR, J. M. (2002). A semi-parametric accelerated failure time cure model. Statistics in medicine 21, 21, 3235-3247.

[20] Li, Y. AND Lin, X. (2003). Functional inference in frailty measurement error models for clustered survival data using the simex approach. Journal of the American Statistical Association 98, 461, 191-203.

[21] López-Cheda, A., Cao, R., Jácome, M. A., and Van Keilegom, I. (2017). Nonparametric incidence estimation and bootstrap bandwidth selection in mixture cure models. Computational Statistics \& 8 Data Analysis $105,144-165$.

[22] LU, W. (2008). Maximum likelihood estimation in the proportional hazards cure model. Annals of the Institute of Statistical Mathematics 60, 3, 545574 .

[23] Ma, Y. AND Yin, G. (2008). Cure rate model with mismeasured covariates under transformation. Journal of the American statistical association 103, 482, 743-756.

[24] Mizoi, M. F., Bolfarine, H., and Pedroso-De-Lima, A. C. (2007). Cure rate model with measurement error. Communications in StatisticsSimulation and Computation 36, 1, 185-196.

[25] Müller, U. U. and Van Keilegom, I. (2019). Goodness-of-fit tests for the cure rate in a mixture cure model. Biometrika 106, 1, 211-227.

[26] Musta, E., Patilea, V., and Van Keilegom, I. (2020). A presmoothing approach for estimation in mixture cure models. arXiv:2008.05338.

[27] Nakamura, T. (1990). Corrected score function for errors-in-variables models: Methodology and application to generalized linear models. Biometrika 77, 1, 127-137.

[28] Parsa, M. and Van Keilegom, I. (2020). Accelerated failure time vs cox proportional hazards mixture cure models: David vs Goliath? Submitted.

[29] Patilea, V. and Van Keilegom, I. (2020). A general approach for cure models in survival analysis. Annals of Statistics 48, 4, 2323-2346.

[30] Peng, Y. And Dear, K. B. (2000). A nonparametric mixture model for cure rate estimation. Biometrics 56, 1, 237-243.

[31] Robertson, T., Wright, F. T., And Dykstra, R. L. (1988). Order Restricted Statistical Inference. Wiley Series in Probability and Mathematical Statistics, John Wiley and Sons, Chichester.

[32] Sposto, R. (2002). Cure model analysis in cancer: an application to data from the children's cancer group. Statistics in medicine 21, 2, 293-312.

[33] Stefanski, L. A. (1989). Unbiased estimation of a nonlinear function of a normal mean with application to measurement error models. Communi- 
cations in Statistics-Theory and Methods 18, 12, 4335-4358.

[34] Stringer, S., Denys, D., Kahn, R. S., And Derks, E. M. (2016). What cure models can teach us about genome-wide survival analysis. Behavior genetics 46, 2, 269-280.

[35] Sy, J. P. and Taylor, J. M. (2000). Estimation in a Cox proportional hazards cure model. Biometrics 56, 1, 227-236.

[36] VAn Der VAart, A. W. AND Wellner, J. A. (1996). Weak convergence and empirical processes. Springer Series in Statistics. Springer-Verlag, New York. With applications to statistics.

[37] Wang, C., Hsu, L., Feng, Z., and Prentice, R. L. (1997). Regression calibration in failure time regression. Biometrics, 131-145.

[38] Wycinka, E. And JuRkiewicz, T. (2017). Mixture cure models in prediction of time to default: comparison with logit and Cox models. In Contemporary Trends and Challenges in Finance. Springer, 221-231.

[39] Xu, J. And Peng, Y. (2014). Nonparametric cure rate estimation with covariates. Canadian Journal of Statistics 42, 1, 1-17.

[40] Yilmaz, Y. E., Lawless, J. F., Andrulis, I. L., and Bull, S. B. (2013). Insights from mixture cure modeling of molecular markers for prognosis in breast cancer. Journal of clinical oncology 31, 16, 2047-2054. 